\title{
Diffraction analysis of nonuniform stresses in surface layers: Application to cracked TiN coatings chemically vapor deposited on Mo
}

\author{
W. G. Sloof, B. J. Kooi, R. Delhez, Th.H. de Keijser, and E. J. Mittemeijer \\ Laboratory of Materials Science, Delft University of Technology, Rotterdamseweg 137, 2628 AL Delft, \\ The Netherlands
}

(Received 1 August 1994; accepted 13 October 1995)

\begin{abstract}
Variations of residual stresses in layers on substrates can occur in directions parallel and perpendicular to the surface as a result of compositional inhomogeneity and/or porosity or cracks. Diffraction methods to evaluate such stress variations are presented. Comparison of the experimental value for the stress with a calculated value of the "diffraction-averaged stress," on the basis of a model for the local stresses, proved to be a useful method of stress analysis. It is shown that a direct evaluation of occurring stress-depth profiles is less practical. The method of stress analysis proposed, is applied to chemically vapor deposited TiN coatings on Mo substrates. In these coatings a large tensile stress parallel to the surface develops during cooling from the deposition temperature, due to difference in thermal shrink between coating and substrate. As a result of the cooling-induced stress, cracking of the coating occurs. The mesh width of the crack pattern allows determination of the fracture-surface energy and the fracture toughness of the coating material. Conceiving the cracked coatings as assemblies of freestanding columns, and assuming full elastic accommodation of the thermal mismatch at the column/substrate interface, the stress variations in the coating are calculated. On this basis the diffraction-averaged stress and the depth profile of the laterally averaged stress can be predicted accurately for the cracked TiN layers.
\end{abstract}

\section{INTRODUCTION}

The presence of residual stresses in materials can largely govern their properties. ${ }^{1-5}$ This in particular holds for thin films and coatings. Comprehensive knowledge is not available on the development and relaxation of stresses in layer/substrate assemblies. The case normally considered involves a homogeneous layer subjected to a laterally constant and depth independent stress. ${ }^{6,7}$ However, the occurrence of compositional inhomogeneity, porosity, and cracks induces residual stress variations in directions parallel and perpendicular to the surface of the layer. The treatment for the (x-ray) diffraction analysis of a composition-depth profile is presented in Refs. 8-10. The present paper deals with stress variations in a thin layer. In particular, the attention will be focused on the case of a layer exhibiting loss of internal coherency by the presence of cracks; i.e., the noncoherent layer is composed of entities that more or less are disconnected with respect to each other, but are strictly (coherently) bonded to the joint substrate at the layer/substrate interface, where the stress-inducing misfit is imposed. The stress distributions in the layer will be described by conceiving the layer as a system of freestanding columns. It will be shown how (x-ray) diffraction analysis of the stress variations in the columns can be performed.
The analysis will be applied to TiN coatings on Mo substrates. TiN coatings are widely used for their high resistance to wear and corrosion. ${ }^{11-14}$ TiN coatings on Mo, in particular, are promising candidates as firstwall materials for a fusion reactor, ${ }^{15}$ because of the low atomic numbers, the high melting point $(3560 \mathrm{~K})$, and a low sputtering yield under ion bombardment.

The TiN coatings investigated $(1-11 \mu \mathrm{m}$ thick) were chemically vapor deposited (CVD) on Mo substrates by reaction of $\mathrm{TiCl}_{4}$ and $\mathrm{NH}_{3}$ in $\mathrm{H}_{2}$ at $973 \mathrm{~K}$. Large residual stresses develop in CVD TiN coatings during cooling from the deposition to room temperature, due to the difference in thermal shrink between coating and substrate. ${ }^{6}$ The tensile stresses became so large that cracks developed in the TiN coatings, bringing about stress relaxation. The complex stress distribution that results for such a cracked layer/substrate assembly has been modeled and will be used to interpret the experimental data.

\section{DIFFRACTION ANALYSIS OF NONUNIFORM STRAINS AND STRESSES}

In coherent and homogeneous surface layers, the state of stress is usually biaxial and the values of the principal components of (residual) stress can be predicted by 
applying simple models (e.g., Refs. 6 and 7). However, produced layers frequently contain irregularities such as pores and cracks. For such internally noncoherent layers, an imposed strain can be taken up by shape changes adjacent to internal free surfaces. Consequently, the orientation and magnitude of the principal stresses depend on the position in the layer.

The local strain $\epsilon_{\phi, \psi}$ in an elastically isotropic solid in a direction $L_{3}$ of the laboratory system given by the Euler angles $\phi$ and $\psi$ with respect to the $S_{1}$ and $S_{3}$ directions, respectively, of the specimen system (see Fig. 1) can be expressed by ${ }^{16}$

$$
\begin{aligned}
\epsilon_{\phi, \psi}= & -\frac{\nu}{E}\left(\sigma_{11}+\sigma_{22}+\sigma_{33}\right)+\frac{1+\nu}{E} \sigma_{33} \\
& +\frac{1+\nu}{E}\left(\sigma_{13} \cos \phi+\sigma_{23} \sin \phi\right) \sin 2 \psi \\
& +\frac{1+\nu}{E}\left(\sigma_{11} \cos ^{2} \phi+\sigma_{22} \sin ^{2} \phi-\sigma_{33}\right. \\
& \left.\quad+\sigma_{12} \sin 2 \phi\right) \sin ^{2} \psi
\end{aligned}
$$

where $\sigma_{i j}(i, j=1,2$, or 3$)$ are the local stress tensor components in the specimen system $S_{i}, E$ is Young's modulus, and $\nu$ is Poisson's ratio. Both the laboratory $L_{i}$ and specimen $S_{i}$ systems (with $i=1,2$, or 3) are spanned by a set of orthonormal basis vectors. For a flat specimen $S_{1}$ and $S_{2}$ are taken parallel to the surface.

In (x-ray) diffraction stress analysis, stresses are derived from experimentally determined values of a number of $\phi$ and $\psi$ settings of the spacing of a particular set of crystallographic planes with indices $H K L$ variable. An experimental value for $\epsilon_{\phi, \psi}$ is obtained according to

$$
\epsilon_{\phi, \psi}^{H K L}=\frac{d_{\phi, \psi}^{H K L}-d_{0}^{H K L}}{d_{0}^{H K L}},
$$

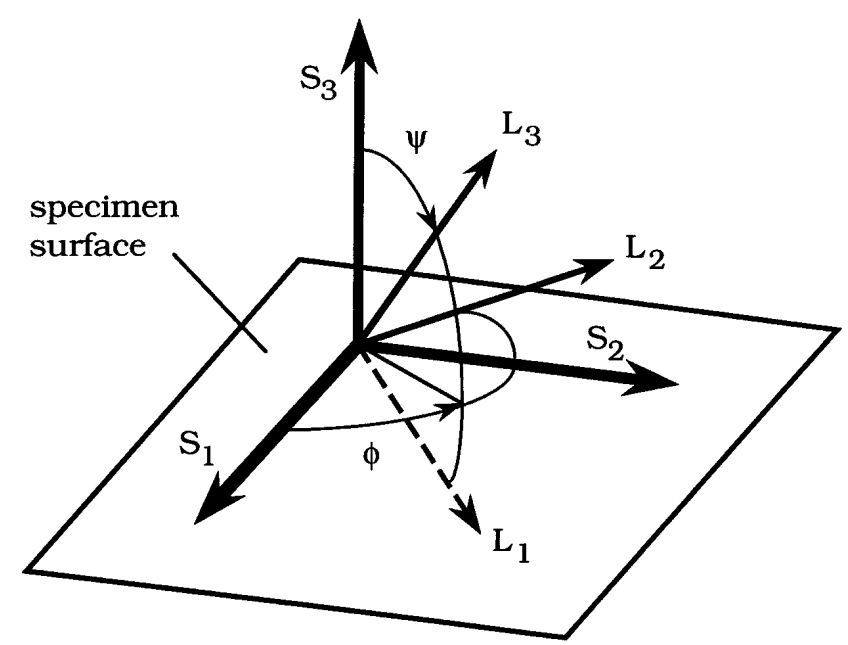

FIG. 1. Definition of the Euler angles $\phi$ and $\psi$ and the orientation of the Cartesian laboratory coordinate system $L_{i}$ with respect to the Cartesian specimen coordinate system $S_{i}(i=1,2$, or 3$)$. where $d$ is the $H K L$ lattice spacing and $d_{0}$ is the strainfree $H K L$ lattice spacing.

The lattice spacing is derived from the recorded diffraction profile (i.e., intensity versus Bragg angle $2 \theta$ ). The recorded intensity stems from the diffracting volume and thus depends on absorption. This absorption effect should be taken into account, if the stress present depends on the depth below the surface. So, the lattice spacing as derived, in fact, represents a diffractionaveraged value, here taken as the intensity weighted average value. Provided that the amount of material averaged over a plane parallel to the surface at any depth $z$ within the diffracting volume is the same, it holds for the $H K L$ lattice spacing at any $\phi$ and $\psi$ (cf. Refs. 8 and 9):

$$
\left\langle d_{\phi, \psi}^{H K L}\right\rangle=\frac{\iint_{V} d_{\phi, \psi}^{H K L} \exp (-\mu \kappa z) d x d y d z}{\iiint_{V} \exp (-\mu \kappa z) d x d y d z},
$$

where $\mu$ denotes the linear absorption coefficient and $\kappa$ is a geometry dependent factor. ${ }^{9}$ The diffracting volume $V$ is defined in a Cartesian coordinate system with axes $X, Y$, and $Z$; see Fig. 2. Note that the $X$ and $Y$ axes are taken parallel with the $S_{2}$ and $S_{1}$ directions, respectively, of the specimen system, and the $Z$ axis is taken in a direction opposite to the $S_{3}$ axis. For a surface layer with thickness $t$ on a substrate material which does not contribute to the diffraction of the considered $H K L$ reflection, it holds that $0 \leqslant z \leqslant t$.

For a powder diffractometer allowing $\psi$ tilt (the $\psi$ axis coincides with the intersection of specimen surface and plane of diffraction), $\kappa$ is given by

$$
\kappa=\frac{2}{\sin \theta \cos \psi},
$$

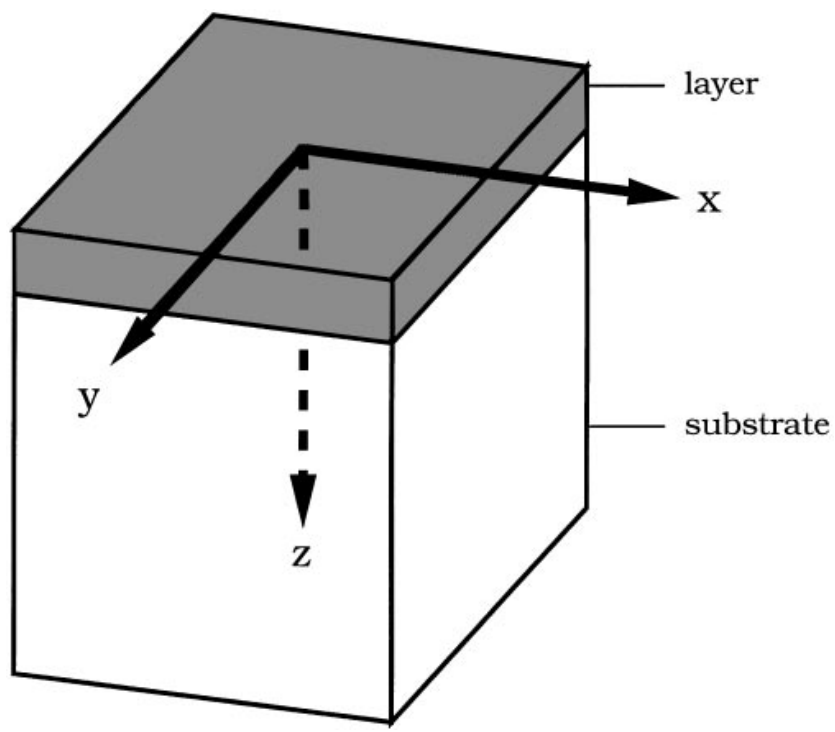

FIG. 2. Definition of auxiliary Cartesian coordinate system (axes $x, y$, and $z$ ) to describe the layer/substrate assembly. 
whereas for a powder diffractometer allowing $\omega$ tilt (the $\omega$ axis coincides with the $\theta$ axis), recognizing that $\omega$ corresponds with $\psi$ in Fig. 1, the relation with $\psi=\omega$ is

$$
\kappa=\frac{2 \sin \theta \cos \psi}{\sin ^{2} \theta-\sin ^{2} \psi} .
$$

In accordance with Eqs. (2) and (3), it follows for the diffraction-averaged strain $\left\langle\epsilon_{\phi, \psi}^{H K L}\right\rangle$ :

$$
\left\langle\epsilon_{\phi, \psi}^{H K L}\right\rangle=\frac{\left\langle d_{\phi, \psi}^{H K L}\right\rangle-d_{0}^{H K L}}{d_{0}^{H K L}},
$$

provided that the strain-free lattice spacing $d_{0}$ is constant within the diffracting volume $V$.

The diffraction-averaged strain is related to the diffraction-averaged stress components [cf. Eq. (1)] by

$$
\begin{aligned}
\left\langle\epsilon_{\phi, \psi}^{H K L}\right\rangle= & -\frac{\nu}{E}\left(\left\langle\sigma_{11}\right\rangle+\left\langle\sigma_{22}\right\rangle+\left\langle\sigma_{33}\right\rangle\right)+\frac{1+\nu}{E}\left\langle\sigma_{33}\right\rangle \\
& +\frac{1+\nu}{E}\left(\left\langle\sigma_{13}\right\rangle \cos \phi+\left\langle\sigma_{23}\right\rangle \sin \phi\right) \sin 2 \psi \\
& +\frac{1+\nu}{E}\left(\left\langle\sigma_{11}\right\rangle \cos ^{2} \phi+\left\langle\sigma_{22}\right\rangle \sin ^{2} \phi\right. \\
& \left.-\left\langle\sigma_{33}\right\rangle+\left\langle\sigma_{12}\right\rangle \sin 2 \phi\right) \sin ^{2} \psi
\end{aligned}
$$

with [cf. Eq. (3)]:

$$
\left\langle\sigma_{i j}\right\rangle=\frac{\iiint_{V} \sigma_{i j} \exp (-\mu \kappa z) d x d y d z}{\iiint_{V} \exp (-\mu \kappa z) d x d y d z} .
$$

It is noted that $\left\langle\sigma_{i j}\right\rangle$ depends on $\psi$ and $\theta$ through $\kappa$ [cf. Eqs. (4a) and (4b)]. To account for elastic anisotropy of the crystals, the macroscopical elastic constants $-\nu / E$ and $(1+\nu) / E$ in Eqs. (1) and (6) are usually replaced by the $H K L$ dependent (x-ray) elastic constants $s_{1}^{H K L}$ and $\frac{1}{2} s_{2}^{H K L}$ (for discussion, see Refs. 17 and 18).

Equation (6) can be simplified considerably as a result of mechanical equilibrium conditions. Consider the stress components as averaged over a plane at depth $z$ below and parallel to the surface. These laterally averaged stresses, $\bar{\sigma}_{i j}$, are defined as:

$$
\bar{\sigma}_{i j}=\frac{\iint_{A} \sigma_{i j} d x d y}{\iint_{A} d x d y},
$$

where $A$ is the area, inside the diffracting volume, of the plane considered at depth $z$ parallel to the surface. ( $A$ is taken independent of $z$.) Obviously, in contrast with $\left\langle\sigma_{i j}\right\rangle, \bar{\sigma}_{i j}$ is independent of $\kappa$ and thus independent of $\psi$ and $\theta$. Then, because the intensity weighting is identical for volume elements at the same depth below the surface, the diffraction-averaged stress [cf. Eq. (7)] can be written as:

$$
\left\langle\sigma_{i j}\right\rangle=\frac{\int \bar{\sigma}_{i j} \exp (-\mu \kappa z) d z}{\int \exp (-\mu \kappa z) d z} .
$$

For a layer of thickness $t: 0 \leqslant z \leqslant t$.

If no external forces act on the body concerned and if the effects of so-called body forces (e.g., gravitation) are negligible, and provided that (a) the distributions of irregularities such as porosity, cracks, lattice defects, etc., which cause variation of local stresses, are random in each plane parallel to the surface, and (b) the distances between these irregularities are small with respect to the lateral dimensions of the diffracting volume, it holds for the laterally averaged stresses at each depth $z$ parallel to the surface that (cf. Appendix A): (i) the component perpendicular to the surface is zero, $\bar{\sigma}_{33}=0$, and (ii) the shear components perpendicular to the surface are zero, $\bar{\sigma}_{13}=\bar{\sigma}_{23}=0$. Thus $\left\langle\sigma_{33}\right\rangle=0$ and $\left\langle\sigma_{13}\right\rangle=\left\langle\sigma_{23}\right\rangle=$ 0 [cf. Eq. (9)], which leads to [cf. Eq. (6)]:

$$
\begin{aligned}
\left\langle\epsilon_{\phi, \psi}^{H K L}\right\rangle=S_{1}^{H K L}\left(\left\langle\sigma_{11}\right\rangle\right. & \left.+\left\langle\sigma_{22}\right\rangle\right) \\
+ & \frac{1}{2} S_{2}^{H K L}\left(\left\langle\sigma_{11}\right\rangle \cos ^{2} \phi+\left\langle\sigma_{22}\right\rangle \sin ^{2} \phi\right. \\
& \left.+\left\langle\sigma_{12}\right\rangle \sin 2 \phi\right) \sin ^{2} \psi .
\end{aligned}
$$

Thus, for a single phase material with spatially changing states of stresses, it follows that the state of stress in terms of diffraction-averaged stresses can be conceived as virtually biaxial, although it should be recognized that the diffraction-averaged stress components depend on $\phi$ and $\psi$. Hence, the state of stress for such a material can be described with "principal" diffraction-averaged stresses by adopting the $S_{1}$ (or $Y$ ) and $S_{2}$ (or $X$ ) axes (cf. Fig. 1) as principal axes.

Often the strain imposed on a surface layer by the substrate is isotropic. Then, for the shear stress $\sigma_{12}$ in the coating it holds at any depth $z$ that $\bar{\sigma}_{12}=0$ (cf. Appendix A). If also the distributions of irregularities and crystal orientation are random or rotationally symmetric with respect to the surface normal, then the laterally averaged stresses/strains are independent of the angle $\phi$. (This independence on $\phi$ can also be achieved by rotation of the specimen around the surface normal during the diffraction measurements.) For such cases Eq. (10) reduces to (omitting the subscript $\phi$ ):

$$
\left\langle\epsilon_{\psi}^{H K L}\right\rangle=2 s_{1}^{H K L}\left\langle\sigma_{/ /}\right\rangle+\frac{1}{2} s_{2}^{H K L}\left\langle\sigma_{/ /}\right\rangle \sin ^{2} \psi
$$

with $\left\langle\sigma_{/ /}\right\rangle=\left\langle\sigma_{11}\right\rangle=\left\langle\sigma_{22}\right\rangle$, where $\left\langle\sigma_{/ /}\right\rangle$is the diffraction-averaged stress parallel to the surface. Recognizing that $\left\langle\sigma_{/ /}\right\rangle$depends on $\psi$ and $\theta$ through $\kappa$ [Eqs. (7) and (9)], plotting $\left\langle\epsilon_{\psi}^{H K L}\right\rangle$ (or $\left\langle d_{\psi}^{H K L}\right\rangle$ ) versus $\sin ^{2} \psi$ does not yield a straight line. The slope of the curve reads:

$$
\begin{gathered}
\frac{\partial\left\langle\epsilon_{\psi}^{H K L}\right\rangle}{\partial \sin ^{2} \psi}=\left(2 s_{1}^{H K L}+\frac{1}{2} s_{2}^{H K L} \sin ^{2} \psi\right) \frac{\partial\left\langle\sigma_{/ \prime}\right\rangle}{\partial \sin ^{2} \psi} \\
+\frac{1}{2} s_{2}^{H K L}\left\langle\sigma_{/ \prime}\right\rangle .
\end{gathered}
$$


In the case of depth independent laterally averaged stresses, thus $\bar{\sigma}_{/ /}$is a constant; obviously $\left\langle\sigma_{/ /}\right\rangle$does not depend on $\psi$ or $\theta$ [cf. Eqs. (7) and 9)]. Then, plotting of $\left\langle\epsilon_{\psi}^{H K L}\right\rangle$ (or $\left\langle d_{\psi}^{H K L}\right\rangle$ ) versus $\sin ^{2} \psi$ results in a straight line, and a value for $\left\langle\sigma_{\| /}\right\rangle$is obtained from its slope $1 / 2 s_{2}^{H K L}\left\langle\sigma_{/ /}\right\rangle$(or $\left.1 / 2 s_{2}^{H K L} d_{0}\left\langle\sigma_{/ /}\right\rangle\right)$.

In the case of depth dependent $\bar{\sigma}_{/ /}$, an accessible value for $\left\langle\sigma_{\| /}\right\rangle$is obtained from the slope of $\left\langle\epsilon_{\psi}^{H K L}\right\rangle$ or $\left\langle d_{\psi}^{H K L}\right\rangle$ versus $\sin ^{2} \psi$ [cf. Eq. (12)] at ${ }^{8}$

$$
\sin ^{2} \psi_{0}=\frac{-2 s_{1}^{H K L}}{\frac{1}{2} s_{2}^{H K L}}
$$

where $\psi_{0}$ indicates the so-called "virtually" strain-free direction $\left[\left\langle\epsilon_{\psi}^{H K L}\right\rangle=0\right.$ for $\psi=\psi_{0}$; cf. Eq. (11)]. Hence, from Eqs. (12) and (13):

$$
\begin{aligned}
\left\langle\sigma_{\| /}\right\rangle_{\psi_{0}} & =\frac{1}{\frac{1}{2} s_{2}^{H K L}}\left(\frac{\partial\left\langle\epsilon_{\psi}^{H K L}\right\rangle}{\partial \sin ^{2} \psi}\right)_{\psi_{0}} \\
& =\frac{1}{\frac{1}{2} s_{2}^{H K L} d_{0}}\left(\frac{\partial\left\langle d_{\psi}^{H K L}\right\rangle}{\partial \sin ^{2} \psi}\right)_{\psi_{0}}
\end{aligned}
$$

or

$$
\left\langle\sigma_{\| /}\right\rangle_{\psi_{0}}=\frac{1}{\frac{1}{2} s_{2}^{H K L}}\langle m\rangle_{\psi_{0}}=\frac{1}{\frac{1}{2} s_{2}^{H K L} d_{0}}\left\langle m_{d}\right\rangle_{\psi_{0}} .
$$

Since here $\bar{\sigma}_{/ /}$depends on depth below the surface, the diffraction-averaged stress $\left\langle\sigma_{\| /}\right\rangle_{\psi_{0}}$ depends on the linear absorption coefficient and the diffraction geometry [cf. Eqs. (4) and (9)]. In the special case that the laterally averaged stress is a linear function of $z$, the diffractionaveraged stress corresponds with the laterally averaged stress at a specified depth, the so-called information depth [cf. Refs. 8, 9].

If no a priori knowledge is available about the depth dependence of the laterally averaged stress, the stress-depth profile can be derived in principle from a set of experimentally determined values of diffractionaveraged stresses. Such a set of data can, for example, be obtained by measurements of different analysis depths by selecting different $H K L$ reflections (i.e., Bragg angles $\theta$ ) or applying different radiations (i.e., different wavelengths and attenuation coefficients $\mu$ ) [cf. Ref. 19]. Also, in the case of a series of layers exhibiting a homologous stress behavior, i.e., the depth profile of the laterally averaged stress for a layer with a thickness $t_{p}$ is equal to the depth profile of the laterally averaged stress in the lower part of a thicker layer with thickness $t_{p+1}\left(t_{p+1}>t_{p}\right)$, the depth profile of the laterally averaged stress of a surface layer can be retrieved straightforwardly from the diffraction-averaged stresses. Such a homologous series may also be obtained from a single layer upon successive removals of sublayers. ${ }^{8-10}$ (Then, in principle, a procedure according to Ref. 20 must be applied to account for the stress relaxation induced by removing the sublayers.)
For layers with a stress-depth profile, the laterally averaged stress is described as a function of the distance $h$ with respect to a common reference plane (viz., the layer/substrate interface). A polynomial of degree $n$ is taken for the laterally averaged stress:

$$
\bar{\sigma}_{/ /} \equiv \bar{\sigma}_{/ /}(h)=\sum_{k=0}^{n} a_{k} h^{k}
$$

where $h=t-z$ and $0 \leqslant h \leqslant t$. The coefficient $a_{0}$ of the polynomial denotes the laterally averaged stress at the interface $(h=0), \bar{\sigma}_{/ /}^{0}$. The coefficients $a_{k}$ of the polynomial can be determined from the above-mentioned set of experimental data: $\left\langle\sigma_{/ /}\right\rangle_{p}$ [with $p=1(1) q$ where $q$ is the number of data $(q \geqslant n)]$.

The laterally averaged stress is related to the diffraction-averaged stress according to Eq. (9). The relation to retrieve the coefficients of the polynomial for the depth profile of the laterally averaged stress from a set diffraction averaged stresses is derived in Appendix B and reads [cf. Eq. (B10)]:

$$
\left\langle\sigma_{/ /}\right\rangle_{p}=\sum_{k=0}^{n} a_{k} T_{p k}
$$

where $T_{p k}$ [cf. Eq. (B8)] depends on the coating thickness $t$, the attenuation coefficient $\mu$, and the diffraction geometry factor $\kappa$, which depends on the Bragg angle $\theta$ (reflection) and the tilt angle $\psi$ [cf. Eqs. (4a) and (4b)].

However, in practice it can be difficult to obtain a comprehensive set of sufficiently divergent data. Then an alternative procedure is the following: development of a model for the state of stress in the layer and calculation of the depth profile of the laterally averaged stress using Eq. (8). Finally, the calculated diffractionaveraged values of the stress can be compared with the experimental values for a set of measurements.

\section{EXPERIMENTAL}

\section{A. Specimen preparation}

The Mo (99.93 wt. \%) substrate material was cut in pieces of $20 \times 20 \times 5 \mathrm{~mm}$ from a rolled strip. Both sides of $20 \times 20 \mathrm{~mm}$ were successively ground using SiC emery paper (final stage 600 mesh), lapped with $9 \mu \mathrm{m}$ diamond slurry, and polished with $6 \mu \mathrm{m}$ and then with $3 \mu \mathrm{m}$ diamond paste. After each preparation step the substrates were ultrasonically cleaned in ethanol. After the final diamond polish, an etching was performed in a solution of $\mathrm{HCl}, \mathrm{HNO}_{3}$, and $\mathrm{HF}$ (concentrated solutions, volume ratio $2: 1: 2$ ).

The substrates were covered with TiN in a horizontal hot-wall reactor (quartz tube inner diameter of $100 \mathrm{~mm}$ ). Prior to the deposition of TiN, the reactor was a few times evacuated and subsequently flushed with a gas mixture of Ar with $10 \mathrm{vol} \% \mathrm{H}_{2}$. Thereafter the substrates 
were annealed in the reactor at $1123 \mathrm{~K}$ for $50 \mathrm{~h}$ in a gas flow of Ar with $10 \mathrm{vol} \% \mathrm{H}_{2}$ to reduce native oxides on the substrate surfaces and to relieve stresses induced by substrate preparation. Then the reactor was stabilized at the deposition temperature and a pressure of $10.0 \pm$ $0.2 \mathrm{kPa}$, using pure $\mathrm{H}_{2}$ gas with a flux of $3 \mathrm{l} / \mathrm{min}$. The chemical vapor deposition of TiN was performed with a gas mixture of $3.0 \mathrm{vol} \% \mathrm{TiCl}_{4}$ and $1.7 \mathrm{vol} \% \mathrm{NH}_{3}$ in $\mathrm{H}_{2}$ with a total flux of $3 \mathrm{l} / \mathrm{min}$. The TiN layers were grown during 2.0, 4.0, and $7.1 \mathrm{~h}$ of deposition at $973 \mathrm{~K}$ in separate runs. The substrates were placed horizontally in the reactor on Mo pillars, such that both sides were covered with TiN. The series A coatings refer to the TiN coatings deposited at the lower side of the substrate and the series B refer to the TiN coatings deposited at the upper side of the substrate. Cooling in the reactor under $\mathrm{H}_{2}$ from the deposition temperature to room temperature took about $5 \mathrm{~h}$.

The parameters used for the deposition of TiN from $\mathrm{TiCl}_{4}$ and $\mathrm{NH}_{3}$, as indicated above, were determined from the results of preceding more or less trial and error experiments. In these experiments the reactant concentrations, pressure, gas flux, etc. were varied, and the coating thicknesses were measured. For these experiments alumina plates were used as substrates. It was found that uniform coatings were obtained at low total gas pressures for low reactant concentrations and a $\mathrm{NH}_{3}$ concentration smaller than the $\mathrm{TiCl}_{4}$ concentration. Under the conditions employed the growth rate is practically independent of the gas flux, implying that surface reaction kinetics and not transport phenomena in the gas phase control the growth rate of the coating.

\section{B. Metallography}

The crack pattern occurring in the TiN coatings was observed in backscattered electron images employing a JEOL JXA 733 electron probe x-ray microanalyzer (see Sec. III.C) using an accelerating voltage of $25 \mathrm{kV}$ and an electron beam current of $10 \mathrm{nA}$. The magnifications applied were calibrated using a grid with a spacing of $10.0 \pm 0.1 \mu \mathrm{m}$.

The morphology of the TiN coatings was investigated from fracture surfaces using a JEOL JSM 840 Scanning Electron Microscope (SEM) operating at an accelerating voltage of $35 \mathrm{kV}$ and an electron beam current of about $30 \mathrm{pA}$. The fracture surfaces were obtained as follows: a small strip of $20 \times 5 \times 1 \mathrm{~mm}$ was cut form a side of the specimen $(20 \times 20 \times 5 \mathrm{~mm}$; see Sec. III. A). At the middle of the strip, a notch was cut in the surface opposite to the one covered with TiN until a separation between the tip of the notch and the TiN coating of about $0.1 \mathrm{~mm}$ was left. Then, the sample was fractured at room temperature.

\section{Composition analysis}

Auger Electron Spectroscopy (AES) was employed for a qualitative assessment of composition-depth profiles. A Perkin-Elmer PHI 4300 Scanning Auger Microprobe (SAM) was used, equipped with a cylindrical mirror analyzer and a PHI 04-300 ion gun. The electron beam was operated at an accelerating voltage of $5 \mathrm{kV}$ and a current of $1.5 \mu \mathrm{A}$. The analyzer-energy resolution was set at $0.6 \%$. Sputtering was performed by scanning over the surface with an $\mathrm{Ar}^{+}$ion beam with an energy of $3.5 \mathrm{keV}$. The sputter rate on TiN amounted to $60 \mathrm{~nm} / \mathrm{min}$. After each sputtering of $1 \mathrm{~min}$ spectra were recorded containing the $\mathrm{C} \mathrm{KL}_{23} \mathrm{~L}_{23}, \mathrm{~N}$ $\mathrm{KL}_{23} \mathrm{~L}_{23}$, O KL $\mathrm{KL}_{23} \mathrm{~L}_{23}, \mathrm{Cl} \mathrm{L}_{3} \mathrm{M}_{23} \mathrm{M}_{23}$, Ti $\mathrm{L}_{3} \mathrm{M}_{23} \mathrm{M}_{23}$, Ti $\mathrm{L}_{3} \mathrm{M}_{23} \mathrm{M}_{45}$, and $\mathrm{Mo}_{4} \mathrm{~N}_{45} \mathrm{~N}_{45}$ characteristic Auger lines. The Auger Peak to Peak Heights (APPH) in the spectra in differential distribution were taken as measures for the atomic concentrations. The $\mathrm{N} \mathrm{KL}_{23} \mathrm{~L}_{23}$ line $(379 \mathrm{eV})$ overlaps with the Ti $\mathrm{L}_{3} \mathrm{M}_{23} \mathrm{M}_{23}$ line $(387 \mathrm{eV})$. Therefore, the APPH of this composite line was taken as a measure for $\mathrm{Ti}+\mathrm{N}$, and the APPH of the $\mathrm{Ti}^{\mathrm{L}} \mathrm{M}_{23} \mathrm{M}_{45}$ line was taken as a measure for Ti. Also, the $\mathrm{Cl} \mathrm{L}_{3} \mathrm{M}_{23} \mathrm{M}_{23}$ line $(181 \mathrm{eV})$ overlaps with the Mo $\mathrm{M}_{45} \mathrm{~N}_{23} \mathrm{~V}$ line $(186 \mathrm{eV})$. However, this did not pose a problem because $\mathrm{Cl}$ and Mo did not occur simultaneously at the same depth in the specimen (see Sec. IV.B).

Electron Probe x-ray Micro Analysis (EPMA) was employed for a quantitative assessment of the overall composition of the TiN coatings. A JEOL JXA 733 electron probe $\mathrm{x}$-ray microanalyzer equipped with four wavelength-dispersive spectrometers and one energydispersive system was used. This instrument is provided with Tracor Northern TN 5500 and TN 5600 systems for instrument control, data acquisition, and analysis. A beam of $10 \mathrm{keV}$ electrons and a current of $30 \mathrm{nA}$ was used. The intensities of the $\mathrm{TiK}_{\alpha}, \mathrm{MoL}_{\alpha}$, and $\mathrm{Cl} \mathrm{K}_{\alpha}$ radiations were measured using the wavelength-dispersive spectrometers. The intensities of these radiations were also measured from Ti, Mo, and $\mathrm{Na}_{4} \mathrm{BeAlSi}_{4} \mathrm{O}_{12} \mathrm{Cl}$ (Tugtupite) references, and the corresponding intensity ratios were determined. The $\mathrm{N} \mathrm{K}_{\alpha}$ line $(392 \mathrm{eV})$ overlaps with the Ti LL line $(395 \mathrm{eV})$, which hinders a direct determination of the $\mathrm{NK}_{\alpha}$ intensity. It was verified that no elements other than $\mathrm{Ti}, \mathrm{Mo}, \mathrm{Cl}$, and $\mathrm{N}$ were present (detectability limit: $\sim 0.1 \mathrm{wt}$. \%). Now, taking the nitrogen content as the balance to accomplish $100 \%$, the modified $\Phi(\rho z)$ approach $^{21}$ was applied to determine the $\mathrm{Ti}, \mathrm{Mo}$, and $\mathrm{Cl}$ contents from the above-indicated intensity ratios.

\section{X-ray diffractometry}

$\mathrm{X}$-ray diffraction measurements were performed using $\omega$ diffractometers (Siemens type F and D500). Both diffractometers are equipped with a curved graphite 
monochromator in the diffracting beam. During the measurements the specimens were rotated around the surface normal. The temperature was controlled within $0.5 \mathrm{~K}$ at about $295 \mathrm{~K}$.

For phase identification and texture characterization, diffractograms were recorded in the Bragg-Brentano geometry from 20 to $160^{\circ} 2 \theta$ with $0.5^{\circ} 2 \theta$ step size using $\mathrm{Cu} \mathrm{K}_{\alpha}$ radiation.

For TiN coating-thickness determination, the $\{200\}-$ and $\{400\}-\mathrm{Cu} \mathrm{K}{ }_{\alpha}$ reflections of the Mo substrate were used both in the presence and absence of the coating. The coating thickness $t$ can then be calculated according to

$$
t=-\frac{\sin \theta_{2}}{2 \mu} \ln \frac{\left(I_{1} / I_{2}\right)_{t}}{\left(I_{1} / I_{2}\right)_{t=0}},
$$

where $\theta_{2}$ denotes the Bragg angle of the second-order reflection of the substrate (here $\{400\}-\mathrm{CuK} \mathrm{K}_{\alpha}$ ), $\mu$ indicates the linear absorption coefficient of the coating material, and $I_{1}$ and $I_{2}$ represent the integrated intensity of the first- and second-order reflections, respectively, of the covered $(t)$ and uncovered $(t=0)$ substrates. This method for the determination of the coating thickness is independent of differences in microstructure (i.e., texture, microstrain, and crystallite size as exhibited in the line-profile broadening) between the various substrates.

For stress measurement and strain-free latticespacing determination, the $\{531\}-\mathrm{Cu} \mathrm{K} \beta$ reflection of TiN was used. The choice of this reflection was mainly based on the constraints of (i) minimal overlap with adjacent reflections, (ii) large $2 \theta$ values $\left(\sim 152^{\circ}\right)$ to obtain a high accuracy in the determination of lattice spacings and to be able to reach a large specimen tilt in the $\omega$ diffractometers ( $\omega$ corresponds with $\psi$ in Fig. 1), and (iii) a relatively large analysis depth, realized by the combination of a relatively low linear absorption coefficient of TiN using $\mathrm{CuK}_{\beta}$ radiation $\left(\mu=645.6 \mathrm{~cm}^{-1}\right)$ with a large Bragg angle $\theta$, to achieve adequate averaging over the coating thickness (cf. Sec. V.B). The reflection indicated was measured at tilt angles corresponding with steps of 0.1 on a $\sin ^{2} \psi$ scale to a maximum of $\sin ^{2} \psi=0.8\left(\omega=63.43^{\circ}\right)$.

The profiles measured were corrected only for (i) the dead time of the counting system and (ii) the angledependencies of Lorentz, polarization, and absorption factors. Thereafter, the peak positions were determined by fitting a parabola to the peak region of the profiles. Systematic (small) errors in the peak positions were eliminated by calibration against a well-defined silicon powder (SRM 640a, see Ref. 22).

\section{DATA EVALUATION AND RESULTS}

For interpretation of the values determined for the stress in the TiN coatings, the morphology, microstructure, and adhesion of these coatings must be considered.
For evaluation of the x-ray diffraction data the composition dependent strain-free lattice spacing and the elastic constants of TiN must be known (cf. Sec. II). In the following first the results obtained on structural characteristics and properties of the present TiN coatings will be discussed, and then the results of the x-ray diffraction stress analysis will be presented.

\section{A. Crack pattern, adhesion, and microstructure}

A crack pattern [see Figs. 3(a) and 3(b)] is observed in all the TiN coatings investigated. The cracks appear as hairlines in the surface of the coatings. However, many cracks observed in the TiN coatings of series B show a gap, which suggests that some delamination had occurred at the edges of these cracks. The cracks extend from the surface to the interface with the substrate, as established in cross sections of the layers. The mesh

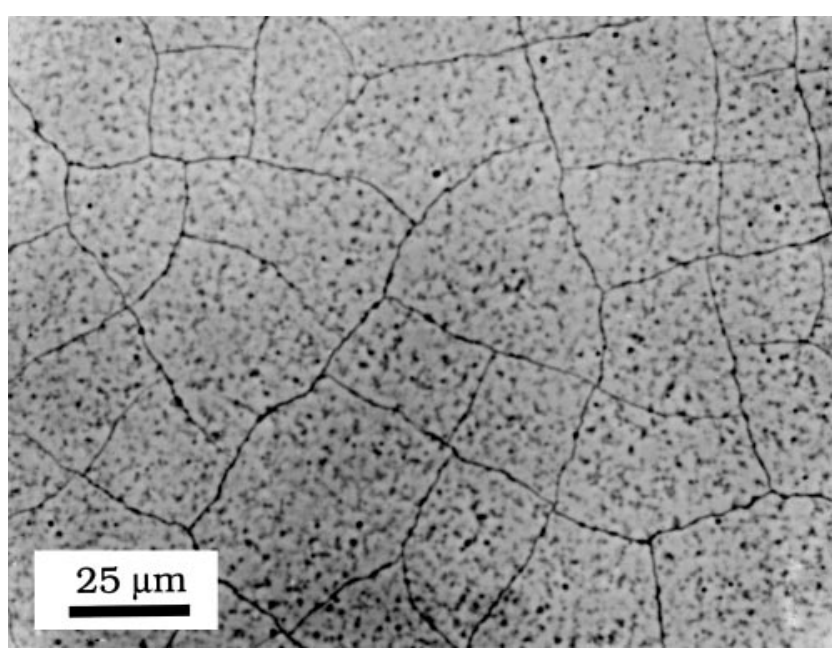

(a)

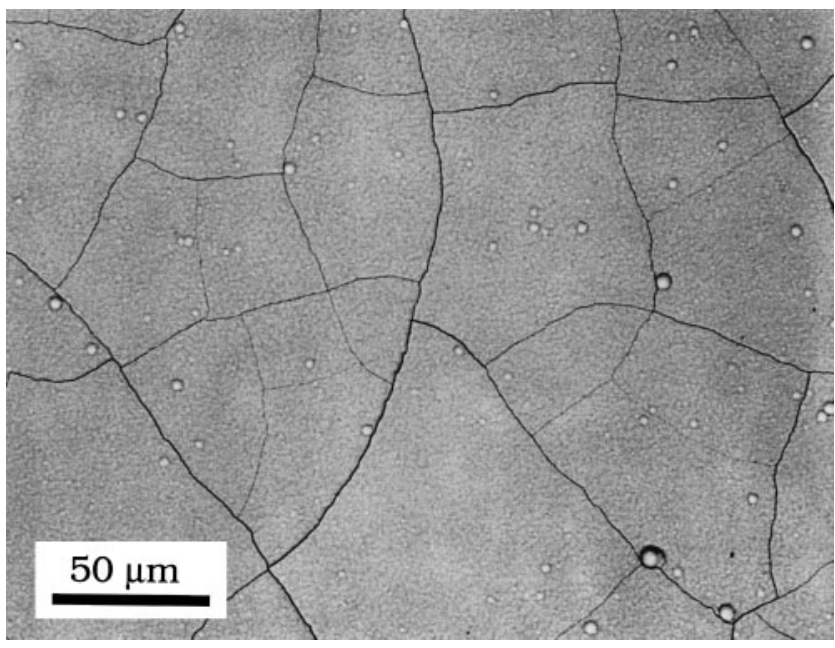

(b)

FIG. 3. Crack patterns in CVD (7.1 h at $973 \mathrm{~K})$ TiN coatings on Mo substrates of series A (a) and B (b) (backscattered electron images). 
width $s$ of the crack pattern increases with increasing coating thickness. This increase in the mesh width is significantly larger for the series B coatings than for the series A coatings; see Table I. This difference between the two series of TiN coatings can be attributed to a distinct difference in adhesion of coating and substrate between the series A and series B coatings (see above and discussion in Sec. V.A).

The adhesion between the TiN coatings and the Mo substrates was found to be significantly better for series A than for series B. This qualitative observation was obtained from scratching with a diamond stylus across the coating surface; the coatings of series B were easily peeled off the substrate, whereas those of series A were not.

For both series of TiN coatings a columnar microstructure is observed with the column axis perpendicular to the surface. The column morphology of the TiN coatings of series A is needle-like, whereas for series B it is more granular, as can be seen in Fig. 4. In this context it is noted that the TiN coatings of both series exhibit an $\langle 100\rangle$-fiber texture with the fiber axis perpendicular to the surface and with the texture strength larger for series A than for series B. Taking the texture coefficient ${ }^{23}$ of the $\{200\}$ reflection in a diffractogram acquired with $\mathrm{CuK}_{\alpha}$ radiation as a measure for the texture strength, it ranges from 4.5 to 6 for series A and from 3 to 4 for series B.

The difference in microstructure originates from the differences between the growth conditions for the two series of coatings. The observed growth rate for the series A coatings is about $25 \%$ smaller than for the series B coatings. Recognizing that the CVD conditions were such that surface reaction kinetics control the growth rate (cf. Sec. III. A), the difference in growth rate is ascribed to incorporation of TiN particles (which were found in the gas phase and came down on the upper side of the specimens) during growth of the series B coatings.

\section{B. Composition}

Composition-depth profiles obtained by Auger analysis showed that the composition of the coating is practically constant over the coating thickness (see Fig. 5). The composition of the TiN coatings was quantified using EPMA. The results are shown in Table II. Some $\mathrm{Cl}$ is present; $\mathrm{C}, \mathrm{O}$, and Mo are absent (with detectability limit less than 0.1 at. \%). Only at the surface of the TiN coatings a little $\mathrm{C}$ and $\mathrm{O}$ is detectable, due to some surface contamination and oxidation.

\section{Elastic constants}

There is considerable disagreement in the literature regarding values for the elastic constants of TiN. ${ }^{24-29}$ The values reported for Young's modulus, $E$, vary between 250 and $640 \mathrm{GPa}$ and those for Poisson's ratio, $\nu$, vary between 0.2 and 0.3 . The present authors consider these data as unreliable, because the deviations may be due to microstructural artifacts in the investigated sintered material, ${ }^{24}$ which may easily contain residual porosity, and in sputtered material, ${ }^{25-29}$ which usually contains many defects. ${ }^{30}$ The values taken here for $E$ and $\nu$, $429 \mathrm{GPa}$ and $0.19(8)$, respectively, have been calculated from the single-crystal elastic constants of $\mathrm{TiN}_{0.98} 31,32$ by using the Eshelby-Kröner model. ${ }^{33}$ Likewise, the x-ray elastic constants for the $H K L$ reflection have been calculated:

$$
\begin{gathered}
s_{1}^{H K L}=(-0.419-0.215 \Gamma) 10^{-6} \mathrm{MPa}^{-1}, \\
\frac{1}{2} s_{2}^{H K L}=(2.67+0.65 \Gamma) 10^{-6} \mathrm{MPa}^{-1},
\end{gathered}
$$

TABLE I. Diffraction-averaged stress $\left\langle\sigma_{/ /}\right\rangle_{\psi_{0}}$ of cracked TiN coatings chemically vapor deposited onto Mo substrates (deposition temperature $973 \mathrm{~K})$ with coating thickness $t$ and mesh width $s$ of the crack pattern. The morphology of the TiN coatings is conceived as an arrangement

\begin{tabular}{|c|c|c|c|c|c|}
\hline Deposition time (h) & $t(\mu \mathrm{m})$ & $s(\mu \mathrm{m})$ & $L / 2 H(-)$ & $\left\langle\sigma_{/ /}\right\rangle_{\psi_{0}}$ experimental $(\mathrm{MPa})$ & $\left\langle\sigma_{/ /}\right\rangle_{\psi_{0}}$ theoretical $(\mathrm{MPa})$ \\
\hline \multicolumn{6}{|l|}{ a. Series A (973 K) } \\
\hline 2.0 & $1.2 \pm 0.1$ & $8 \pm 2$ & $3.3 \pm 0.8$ & $604 \pm 10$ & 617 \\
\hline 2.0 & $1.5 \pm 0.1$ & $10 \pm 2$ & $3.4 \pm 0.7$ & $619 \pm 10$ & 627 \\
\hline 4.0 & $3.2 \pm 0.1$ & $16 \pm 4$ & $2.5 \pm 0.6$ & $457 \pm 10$ & 488 \\
\hline 4.0 & $3.4 \pm 0.1$ & $18 \pm 4$ & $2.6 \pm 0.6$ & $506 \pm 10$ & 487 \\
\hline 7.1 & $5.7 \pm 0.1$ & $25 \pm 5$ & $2.2 \pm 0.4$ & $298 \pm 10$ & 379 \\
\hline 7.1 & $8.7 \pm 0.1$ & $30 \pm 5$ & $1.7 \pm 0.3$ & $212 \pm 10$ & 263 \\
\hline \multicolumn{6}{|l|}{ b. Series B (973 K) } \\
\hline 2.0 & $1.9 \pm 0.1$ & $20 \pm 4$ & $5.3 \pm 1.0$ & $714 \pm 10$ & 743 \\
\hline 2.0 & $2.5 \pm 0.1$ & $21 \pm 3$ & $4.3 \pm 0.6$ & $733 \pm 10$ & 696 \\
\hline 4.0 & $4.5 \pm 0.1$ & $33 \pm 5$ & $3.7 \pm 0.5$ & $446 \pm 10$ & 615 \\
\hline 4.0 & $4.9 \pm 0.1$ & $30 \pm 5$ & $3.1 \pm 0.5$ & $233 \pm 10$ & 556 \\
\hline 7.1 & $10.1 \pm 0.1$ & $45 \pm 6$ & $2.3 \pm 0.3$ & $24 \pm 10$ & 379 \\
\hline 7.1 & $10.4 \pm 0.1$ & $52 \pm 8$ & $2.5 \pm 0.4$ & $60 \pm 10$ & 419 \\
\hline
\end{tabular}
of freestanding columns (see discussion in Sec. V. A) with column geometry factor $L / 2 H$, which is taken equal to $s / 2 t$. 


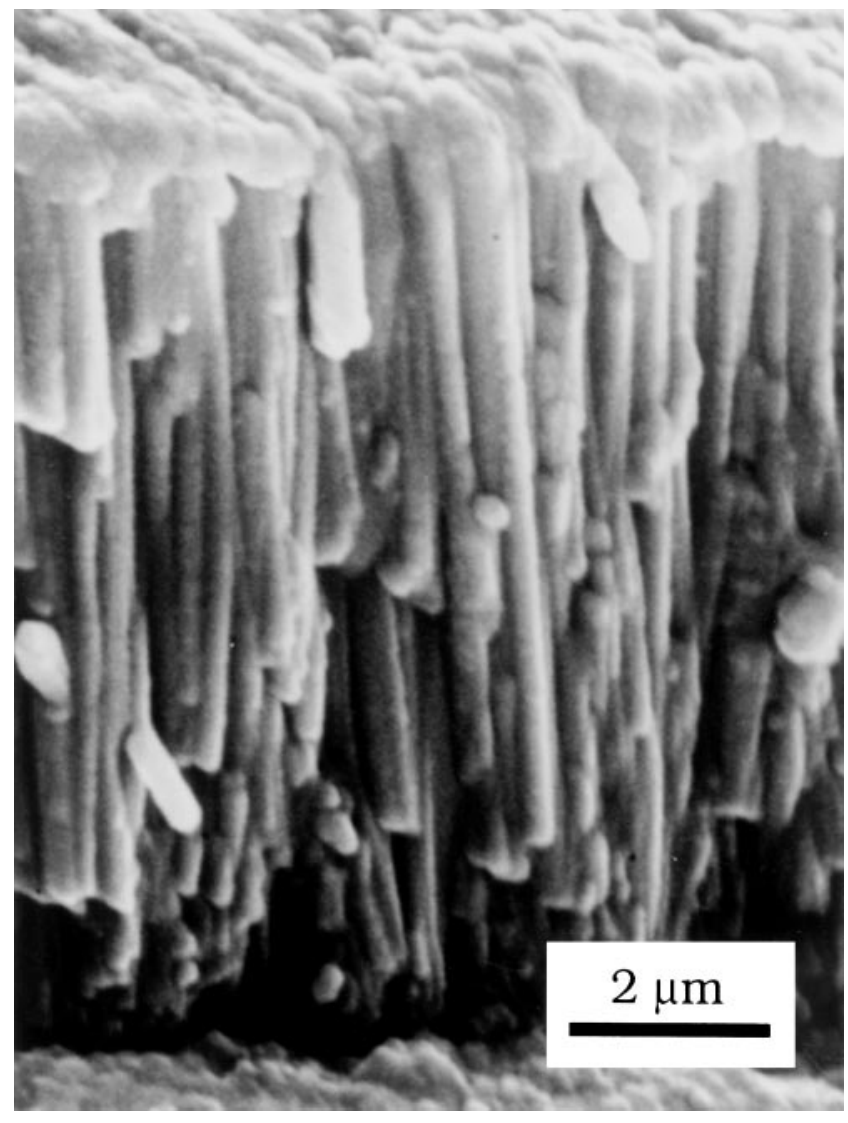

(a)

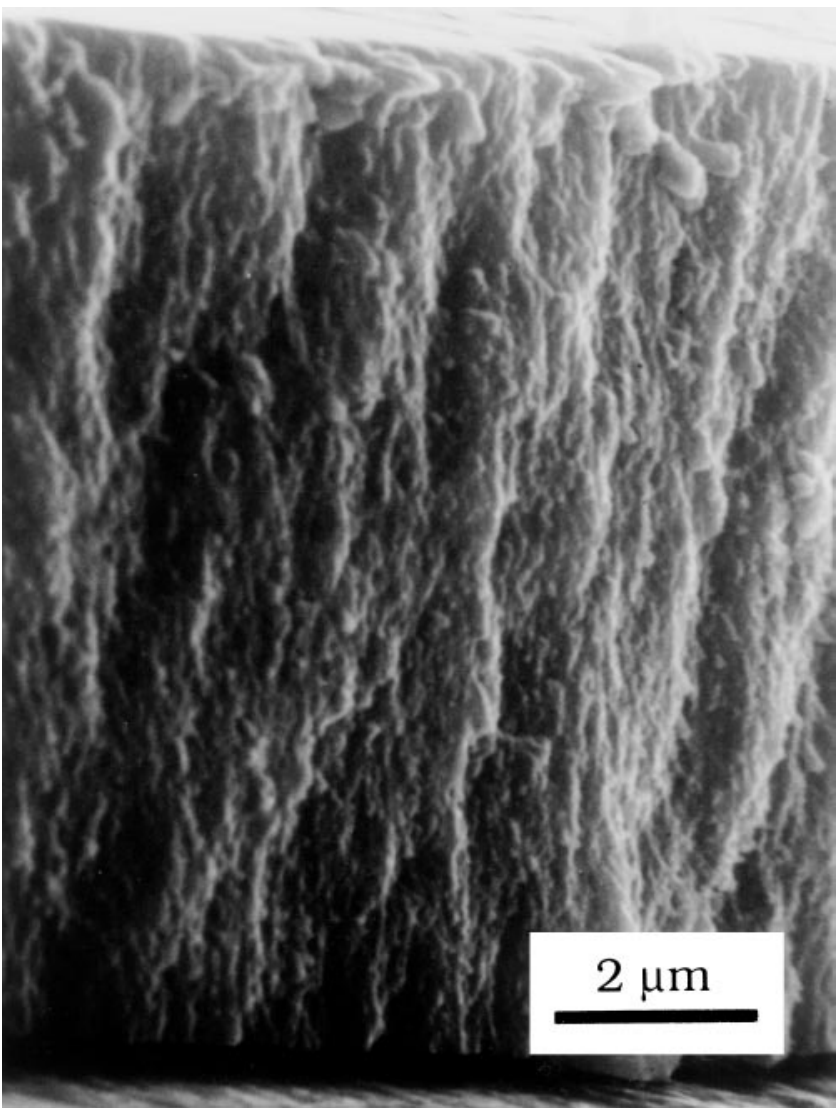

(b)

FIG. 4. Microstructure of CVD (7.1 h at $973 \mathrm{~K}$ ) TiN coatings on Mo substrates of series A (a) and B (b) (secondary electron images of cross sections).

where

$$
\Gamma=\left(H^{2} K^{2}+K^{2} L^{2}+L^{2} H^{2}\right) /\left(H^{2}+K^{2}+L^{2}\right)^{2} .
$$

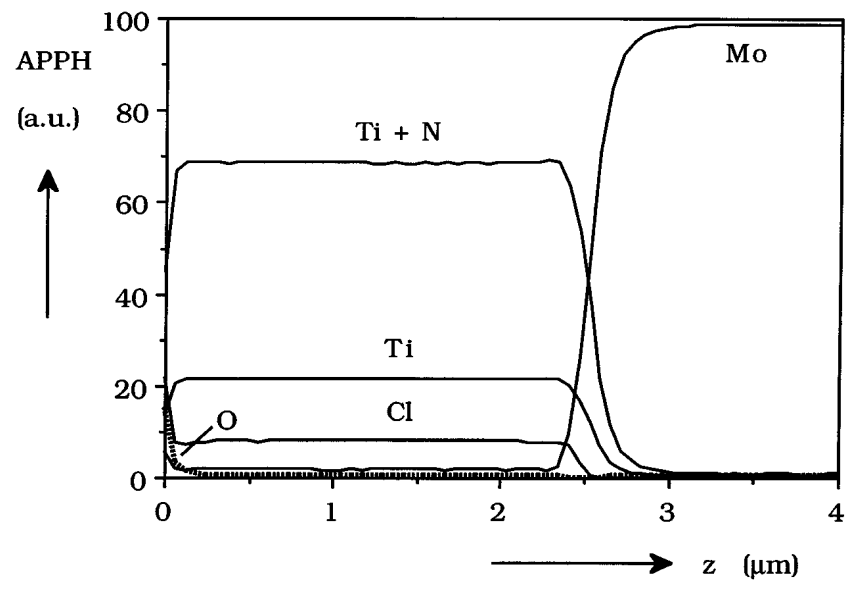

FIG. 5. Normalized Auger peak to peak height (APPH) in arbitrary units (a.u.) versus depth $z$ of a CVD $(2 \mathrm{~h}$ at $973 \mathrm{~K})$ TiN coating on a Mo substrate of series B (Auger depth profiling).
These calculated values for the elastic constants for TiN correspond very well with the elastic constants reported for $\mathrm{TiC}^{7}$ as may be expected because of the chemical and crystallographic similarity of these compounds.

An experimental value for Poisson's ratio can in principle be obtained from the common point of intersection of a set of experimental (x-ray diffraction) curves of $\left\langle d_{\psi}^{H K L}\right\rangle$ versus $\sin ^{2} \psi$ data of TiN coatings of different internal stress, but of the same composition (i.e., of identical strain-free lattice spacing $d_{0}$ ). An example of such a set of data is given in Fig. 6. The common point of intersection provides a value for the strain-free lattice spacing and a value for $\sin ^{2} \psi_{0}$ [cf. Eqs. (11) and (13)].

TABLE II. Composition and (strain-free) lattice parameter, $a$, of TiN coatings chemically vapor deposited at $973 \mathrm{~K}$ onto Mo substrates.

\begin{tabular}{cccccr}
\hline \hline $\begin{array}{c}\text { Specimen } \\
\text { series }\end{array}$ & $\begin{array}{c}\mathrm{Ti} \\
(\text { at. \%) }\end{array}$ & $\begin{array}{c}\mathrm{N} \\
(\text { at. \%) }\end{array}$ & $\begin{array}{c}\mathrm{Cl} \\
(\text { at. \%) }\end{array}$ & $\begin{array}{c}\mathrm{N} / \mathrm{Ti} \\
(-)\end{array}$ & $\begin{array}{c}a \\
(\mathrm{~nm})\end{array}$ \\
\hline $\mathrm{A}$ & 52.7 & 45.5 & 1.87 & 0.86 & 0.42411 \\
$\mathrm{~B}$ & 52.6 & 45.2 & 2.21 & 0.86 & 0.42416 \\
$\cdots$ & \pm 0.5 & \pm 0.5 & \pm 0.5 & \pm 0.2 & \pm 0.00002 \\
\hline \hline
\end{tabular}




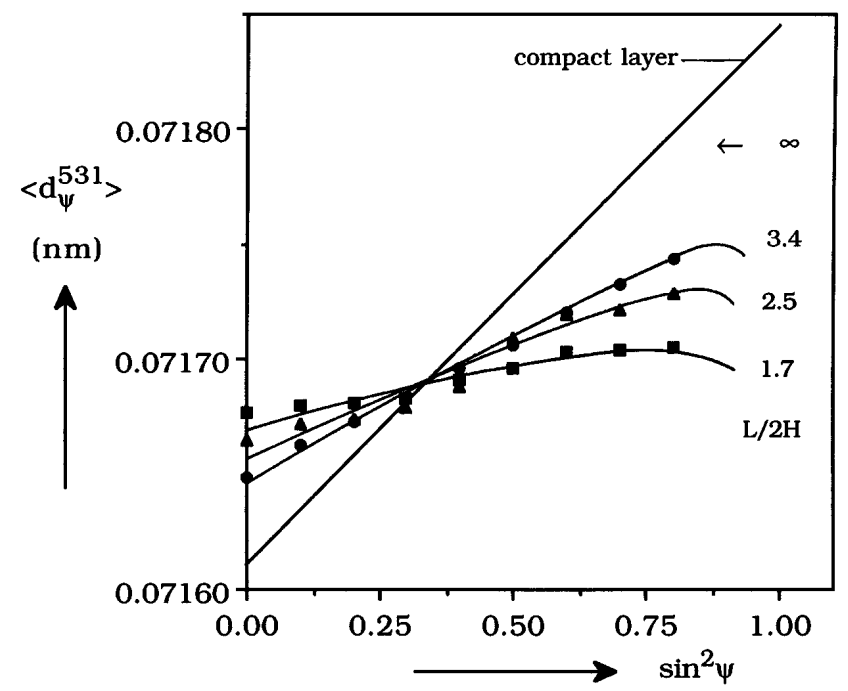

FIG. 6. Experimentally (dots) determined and theoretically (full lines) predicted values of $\left\langle d_{\psi}^{531}\right\rangle$ plotted as a function of $\sin ^{2} \psi$ for CVD [series A $(973 \mathrm{~K})$ ] TiN coatings on Mo substrates for various values of the column geometry factor $L / 2 H$ (cf. Sec. V. A and Table I). The theoretical curves have been calculated without adaptation of the model parameters; i.e., no fitting has been performed.

For elastically isotropic materials it holds that $\sin ^{2} \psi_{0}=$ $2 \nu /(1+\nu)$ [cf. Eq. (13) and below Eq. (7)]. This result may also be applied to elastically anisotropic materials of cubic crystal symmetry, because the strain-free direction of these materials appears to be virtually independent of elastic anisotropy [e.g., for TiN this can be verified easily; cf. Eqs. (18a) and (18b)]. Consequently, a value for $\nu$ can be calculated. To achieve averaging over all data available, the analysis is performed here as follows; see Fig. 7. The intercept at $\sin ^{2} \psi=0$ of the tangent

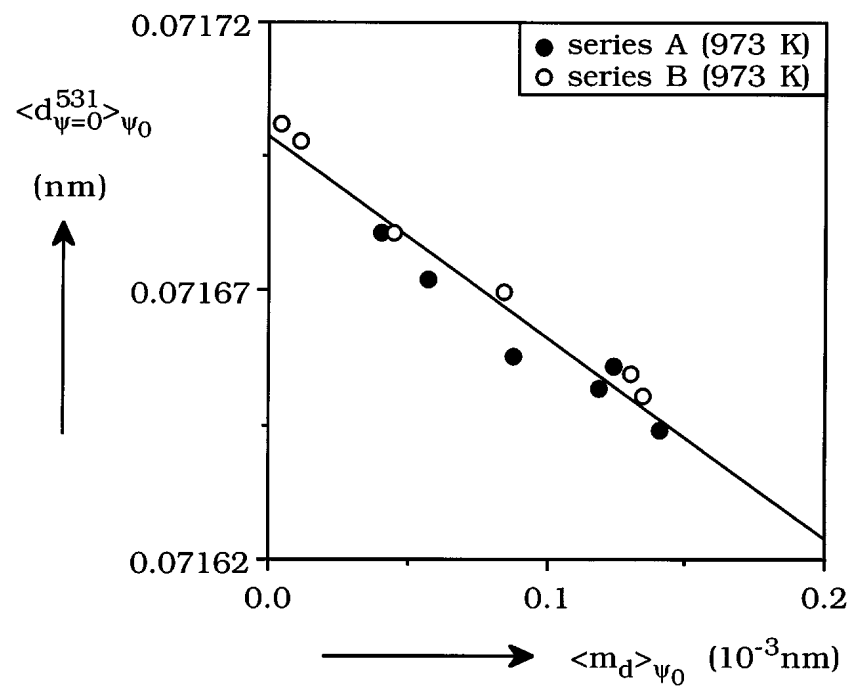

FIG. 7. Plot of $\left\langle d_{\psi=0}^{531}\right\rangle_{\psi_{0}}$ versus $\left\langle m_{d}\right\rangle_{\psi_{0}}$, i.e., the intercept at $\sin ^{2} \psi=0$ of the tangent line to the $\left\langle d_{\psi}^{531}\right\rangle$ versus $\sin ^{2} \psi$ curves (cf. Fig. 6) at the point of common intersection (i.e., at about $\sin ^{2} \psi_{0}$ ) versus the slope of the same tangent line, for the CVD (at $973 \mathrm{~K}$ ) TiN coatings on Mo substrates. to the $\left\langle d_{\psi}^{H K L}\right\rangle$ versus $\sin ^{2} \psi$ curve (cf. Fig. 6) at the common point of intersection $\left(\left\langle d_{\psi=0}^{H K L}\right\rangle_{\psi_{0}}\right)$ is plotted versus the corresponding slope $\left\langle m_{d}\right\rangle_{\psi_{0}}\left[=\frac{1}{2} s_{2}^{H K L} d_{0}\left\langle\sigma_{/ /}\right\rangle_{\psi_{0}}\right.$; cf. Eq. (14)]. This type of plot has been proposed in Ref. 8. For the straight line through these data points, it holds

$$
\left\langle d_{\psi=0}^{H K L}\right\rangle_{\psi_{0}}=d_{0}-\sin ^{2} \psi_{0}\left\langle m_{d}\right\rangle_{\psi_{0}} .
$$

Hence, the slope of the straight line fitted through the plotted data provides an average value for $\sin ^{2} \psi_{0}$ and the intersection of the line with the ordinate yields an average value for $d_{0}$. Because of the presence of $\left\langle m_{d}\right\rangle_{\psi_{0}}$ in Eq. (19), it seems that this suggested procedure presupposes knowledge of $\psi_{0}$ or $\sin ^{2} \psi_{0}$ (instead of delivering a value for $\psi_{0}$ or $\sin ^{2} \psi_{0}$ ). However, in many cases (including the present case) the $\left\langle d_{\psi}^{H K L}\right\rangle$ versus $\sin ^{2} \psi$ plot shows practically a straight line, and for $\left\langle m_{d}\right\rangle_{\psi_{0}}$ in Eq. (19) the average slope $\left\langle m_{d}\right\rangle$ can be substituted. Thus it is obtained: $\sin ^{2} \psi_{0}=0.375 \pm$ 0.030 and $\nu=0.23 \pm 0.02$ of TiN. (For $d_{0}$ see next section.) This experimental value for Poisson's ratio is close to the value calculated above from the single crystal elastic constants; the calculated value will be used in the following.

\section{Lattice parameter}

The lattice parameter for each of the TiN coatings is obtained from the strain-free lattice spacing $d_{0}$, as determined by interpolation in the $\left\langle d_{\psi}^{531}\right\rangle$ versus $\sin ^{2} \psi$ plot at the strain-free direction [cf. Eq. (13)] calculated with the elastic constants (see Sec. IV.C). [It should be noted that the procedure employed here allows that $d_{0}$ is different for all coatings, in contrast with the alternative procedure involving the application of Eq. (19) which presupposes that the $d_{0}$ is the same for all coatings.] The average lattice parameter for each series of TiN coatings is presented in Table II. The values obtained in this way agree with the values for the lattice parameter determined for some pulverized (and hence free of residual macrostress) TiN coatings.

The lattice parameter of TiN depends on the N/Ti atomic ratio, ${ }^{34}$ which according to the $\mathrm{Ti}-\mathrm{N}$ phase diagram $^{35}$ can vary between 0.57 and 1 . For the present coatings the composition (see Sec. IV.B) and thus the lattice parameter is constant over the thickness of the coating. Because the $\mathrm{N} / \mathrm{Ti}$ atomic ratio is the same for all coatings of each of the series A and B, the significantly larger lattice parameter for the series $\mathrm{B}$ coatings is ascribed to their higher $\mathrm{Cl}$ content. $\mathrm{Cl}$ dissolved in TiN increases the lattice parameter, because the atomic radius of $\mathrm{Cl}$ is larger than that of $\mathrm{Ti}$ and of $\mathrm{N} .{ }^{36}$

\section{E. Stresses}

The diffraction method outlined in Sec. II for the analysis of stress-depth profiles implies that the strain- 
free lattice spacing is constant within the diffracting volume. For the present TiN coatings this condition is met, because the composition (see Sec. IV.B) and thus the lattice parameter (see Sec. IV.D) do not vary as a function of depth. [If the strain-free lattice spacing would vary with depth (e.g., due to the presence of a composition-depth profile in the coatings), then an additional curvature in the $\left\langle d_{\psi}^{H K L}\right\rangle$ versus $\sin ^{2} \psi$ curve is invoked (because the information depth changes as a function of $\sin ^{2} \psi$ ), which then could erroneously be interpreted as a stress (so-called "ghost" stress $^{8}$ )].

The stress $\left\langle\sigma_{\| /}\right\rangle_{\psi_{0}}$ is determined by taking the slope of $\left\langle d_{\psi}^{H K L}\right\rangle$ versus $\sin ^{2} \psi$ at $\sin ^{2} \psi_{0}$. In this procedure the term between brackets in Eq. (12) is zero, and the effects of stress variation with depth on the curvature of $\left\langle d_{\psi}^{H K L}\right\rangle$ as a function of $\sin ^{2} \psi$ are avoided. The experimentally determined curves of $\left\langle d_{\psi}^{H K L}\right\rangle$ versus $\sin ^{2} \psi$ derived from the $\{531\}-\mathrm{CuK} \mathrm{K}_{\beta}$ reflection of TiN show practically no curvature (see Fig. 6). This does not necessarily imply that the stress in the coatings does not appreciably change with depth. The variation of the information depth (see Sec. II) as a function of $\sin ^{2} \psi$ for the applied range of tilt angle $\psi$ can be too small with respect to the stress gradient occurring in the TiN coatings. It will be shown in Sec. V.B that this is the case here. In addition to the experimentally determined $\left\langle d_{\psi}^{H K L}\right\rangle$ versus $\sin ^{2} \psi$ curves, theoretically predicted curves (see Sec. V.B) are already shown in Fig. 6. It follows that the deviation from straight-line behavior is large at high $\omega$-tilt angles, which are beyond the range of tilt angles employed in the experiments (cf. Sec. III.D). Thus, in the present case it is allowed to use the slope of the straight line fitted through the $\left\langle d_{\psi}^{H K L}\right\rangle$ versus $\sin ^{2} \psi$ data points for the determination of the (x-ray) diffraction-averaged stress or strain.

The results thus obtained are gathered in Table I. Clearly, the x-ray diffraction-averaged stress $\left\langle\sigma_{/ /}\right\rangle_{\psi_{0}}$ decreases with increasing coating thickness. Note that this behavior is opposite to that observed for the mesh width $s$ of the crack pattern (cf. Sec. IV. A).

In the next section the results of the stress analysis will be explained quantitatively.

\section{DISCUSSION}

\section{A. Stress relaxation, cracking, and debonding}

It is likely that the residual stresses in the TiN coatings do not originate from the growth (CVD) process, ${ }^{6}$ but originate from the difference in thermal shrink between coating and substrate experienced on cooling from the deposition temperature to room temperature. In the absence of delamination, the difference in thermal shrink has to be bridged at the coating/substrate interface. The TiN coatings are very thin as compared with the substrates. Then, according to a simple model based on elastic accommodation of strains, mechanical equilibrium, and a homogeneous stress in both coating and relatively thick substrate, ${ }^{6}$ it follows that the difference in thermal shrink between coating and substrate will be predominantly assimilated by the coating. Because the thermal shrink of TiN and Mo is isotropic, it follows from the coefficients of thermal expansion of $\mathrm{TiN}^{37}$ and $\mathrm{Mo}^{38}$ and from the difference between the deposition temperature $(973 \mathrm{~K})$ and room temperature that an isotropic (thermal) strain $\epsilon_{/ /}=(2.2 \pm 0.1) 10^{-3}$ is imposed on the TiN coating at the coating/substrate interface. Then, this strain results in a biaxial state of stress with two equal principal stresses $\left(\sigma_{11}=\sigma_{22} \equiv\right.$ $\left.\sigma_{\|}\right)$in an uncracked TiN coating. The macroscopic stress $\sigma_{/ /}$is simply related to the macroscopic strain $\epsilon_{/ /}$according to $\sigma_{/ /}=\epsilon_{/ /} E /(1-\nu)$. Using $E$ and $\nu$ for TiN (cf. Sec. IV.C), a macroscopic stress of $\sigma_{/ /}=$ $1170 \pm 60 \mathrm{MPa}$ is predicted for uncracked TiN coatings at room temperature. The experimental values for the internal stress/strain in the TiN coatings are significantly smaller (cf. Table I). Apparently, with respect to the stress expected from the difference in thermal shrink, stress relaxation has occurred in the TiN coatings.

The presence of cracks in the coatings (cf. Sec. IV. A) is associated with stress relaxation. Starting with a crack-free coating, during cooling form the deposition temperature a uniform tensile stress parallel to the coating/substrate interface is built up in the TiN coatings. At a certain stage this stress exceeds the fracture strength of the TiN coatings, resulting in the formation of cracks. In general, the fracture strength of brittle materials as TiN depends strongly on macroscopic defects such as pores and surface roughness (cf. Refs. 39 and 40). Plastic yielding of the coatings can be excluded at the temperatures concerned (see the deformation map of $\mathrm{TiC}$ in Ref. 41, which is similar to that for TiN because of the similarity between their physical and mechanical properties).

In view of the crack patterns observed, involving cracks extending from the surface to the interface with the substrate (sec. IV.A), the morphology of the TiN coatings can be conceived as an arrangement of freestanding columns with the column axis perpendicular to the substrate. Then the column width $L$ is taken as the mesh width $s$ of the crack pattern and the column height $H$ as the coating thickness $t$. As compared with the uncracked situation, the stress due to the thermal misfit imposed at the coating/substrate interface is partly relaxed by shape changes of the columns. A schematic picture is given in Fig. 8, illustrating isostrain contours in the columns, assuming that the coating/substrate misfit experienced at the foot of the column is taken up fully elastically. Such triaxial stress/strain distributions can be calculated by a model presented in detail in Ref. 42. (This column model allows calculation of the 


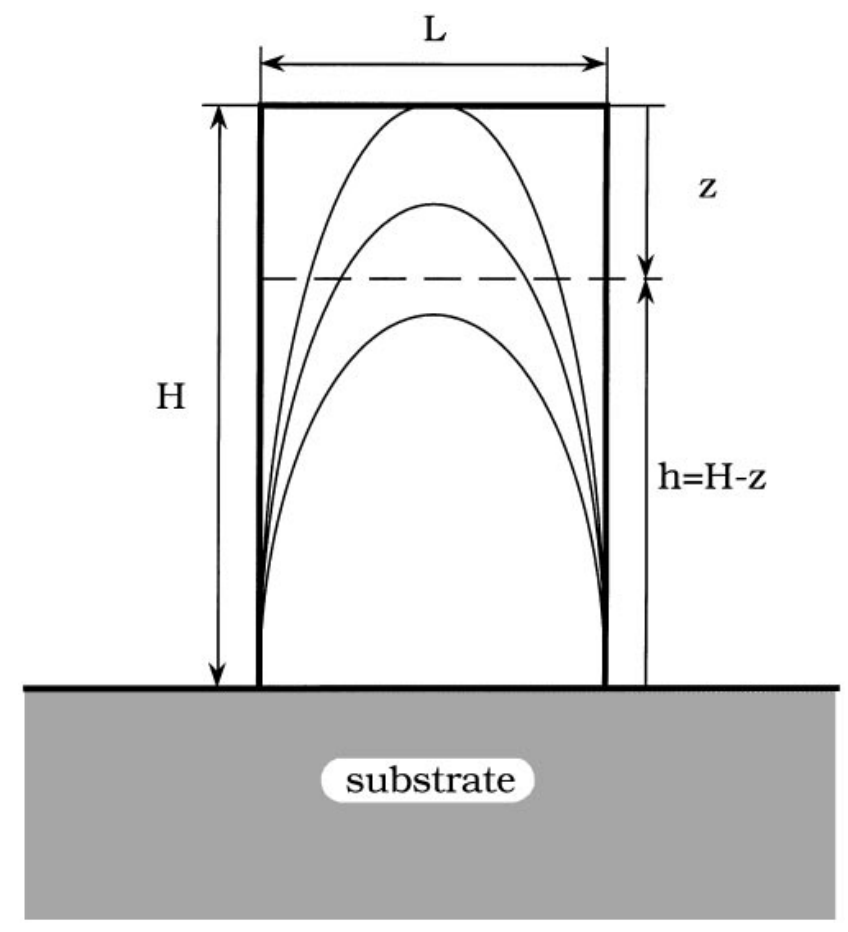

FIG. 8. Schematic presentation of a freestanding column with height $H$ and width $L$ on a substrate. The lines in the column represent isostrain contours; i.e., these lines connect volume elements of equal local strain in directions parallel to the column/substrate interface.

three-dimensional distribution of the thermal stresses induced by a uniform temperature change in a cylindrical column on a substrate. It is a generalization of the two-dimensional description for the problem of thermal stresses in a rectangular plate clamped along an edge as given by Aleck, ${ }^{43}$ with corrections by Blech and Levi ${ }^{44}$ and improved by Blech and Kantor. ${ }^{45}$ ) This model provides the local values of stress and strain components in the columns. They depend, apart from the position in the column and the thermal strain, only on the column geometry defined by $L / 2 H$ and the elastic properties $E$ and $\nu$ of the column material. On the basis of these calculated stresses, a prediction can be made for the stresses which would be determined with diffraction methods (cf. Sec. II). Such predicted and experimentally determined stresses will be discussed in Sec. V. B.

The mesh width of the crack pattern of the series B coatings is larger than the mesh width for the series A coatings of similar thickness (see Table I). The difference between the mesh width of the crack pattern of the series A and B increases with the coating thickness. According to the column model (see above), this would imply that the residual stress for the series B coatings would be larger than for the series A coatings of similar thickness. However, in any case for the thick coatings, the opposite is observed (see Table I). Thus, a process of stress relaxation in addition to cracking of the coating has happened in the concerned coatings of series B. From calculations using the column model, it follows that near the edges at the foot of the column relatively high local shear stresses $\left(\sigma_{12}\right)$ exist parallel to the interface and tensile normal stresses $\left(\sigma_{33}\right)$ exist perpendicular to the interface with the substrate. Both types of stress at the coating/substrate interface stimulate debonding of the coating. Hence, debonding of a coating can be a consequence of cracking of the coating (see also Refs. 46-50). The following arguments indicate that the additional stress relaxation (see above) is due to partial debonding of the series B coatings from the substrate.

(i) The adhesion of the TiN coatings with the substrate is much less for series B than for series A (cf. Sec. IV. A).

(ii) Many cracks in the series B coatings show a gap [cf. Sec. IV. A and Fig. 3(b)], suggesting partial debonding of these coatings from the substrate near the edges of the cracks.

(iii) For the series B coatings (and not for the series A coatings), the residual stress observed is significantly smaller than the stress predicted by the column model, excluding debonding [see Table I and see also Figs. 11(a) and 11(b) discussed in Sec. V. B].

The observed increase of the mesh width of the crack pattern with the thickness of the coatings (cf. Sec. IV. A and Table I) can be explained from the equilibrium described by the balance between decrease of stored elastic strain energy and the increase of fracture-surface energy (Appendix C). It follows from Eq. (C6b) that a linear relation should exist between $L^{2}$ and $H$. The experimental results (Fig. 9) indeed show this behavior for the series A coatings (for the series B coatings, see below). The slope of the straight line provides a value for the fracture-surface energy $\gamma$ and thus the fracture toughness $K_{c}$ of TiN [cf. Eq. (C7)]. Using $E$ and $\nu$ of TiN (cf. Sec. IV.C) and with $m=1$ [cf. Eq. (C3)], it is ob-

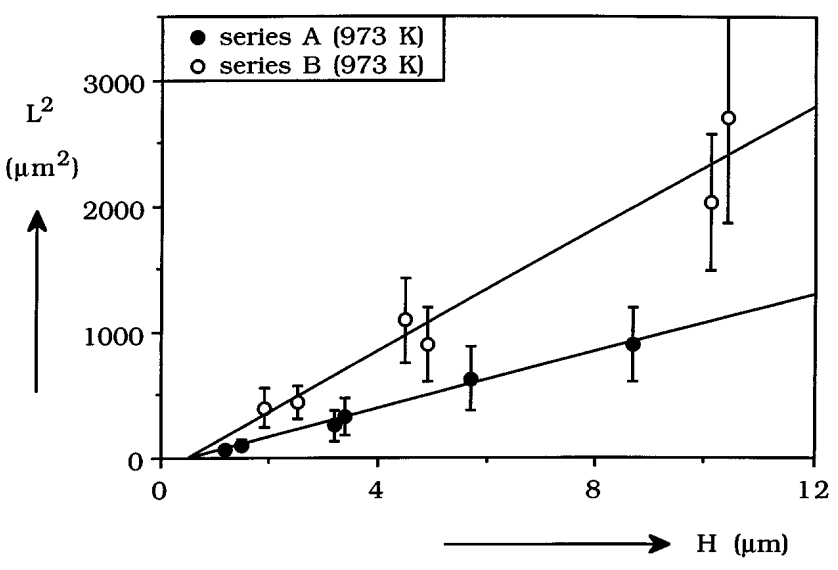

FIG. 9. The square of the column width $L$ (taken as the mesh width $s$ ) versus the column height $H$ (taken as coating thickness $t$ ) for the CVD (at $973 \mathrm{~K}$ ) TiN coatings on Mo substrates (cf. Table I). 
tained that $\gamma=8.2 \mathrm{~J} / \mathrm{m}^{2}$ and $K_{c}=2.6 \mathrm{MPa} \cdot \mathrm{m}^{1 / 2}$. These values correspond well with those reported in Ref. 40 for other brittle materials such as $\mathrm{SiC}, \mathrm{Si}_{3} \mathrm{~N}_{4}$, and $\mathrm{Al}_{2} \mathrm{O}_{3}$. The values reported in Ref. 51 obtained from a TiN coating on cemented carbide (WC-Co) by an indentation method are incorrect because an erroneous value for $E$ was used. Using the value of $E$ preferred here (429 GPa), the values of Ref. 51 can be corrected and become $\gamma=$ $6.1 \mathrm{~J} / \mathrm{m}^{2}$ and $K_{c}=2.3 \mathrm{MPa} \cdot \mathrm{m}^{1 / 2}$, which agree with our results recognizing that values reported for the fracture surface energy of brittle materials in general scatter by a factor of 10 (note that present-day techniques for measuring surface energies of solids have an inherent inaccuracy of about $100 \%$; cf. Refs. 40 and 46).

Now consider the results for the series B coatings (Fig. 9). The slope of a straight line through the data points of $L^{2}$ versus $H$ is significantly larger for the series $\mathrm{B}$ than for the series A coatings. This is in agreement with the idea that in the series A coatings only cracking occurred and that in the series B coatings the cracking is accompanied by delamination. The difference between observed and predicted residual stress for the series B coatings increases with increasing coating thickness [see Table I and see also Fig. 11(b) discussed in Sec. V. B]. Apparently, the extent of debonding and associated stress relaxation increases with increasing coating thickness. This coating thickness dependent behavior can be explained as follows. In the absence of debonding, the (average) amount of elastic strain energy $U_{e}$ per unit of volume stored in the coating depends on the mesh width of the crack pattern and the coating thickness; i.e., depends on the column geometry factor $L / 2 H$; see Appendix C, Eq. (C2) and Fig. 13. If, subsequent to cracking, debonding at the interface between coating and substrate occurs, then the amount of elastic strain energy released by debonding per unit area of the interface, $U_{d}$, is in first-order approximation given by the product of $U_{e}$ and $t$, where $t$ is the coating thickness. For the series B coatings both $U_{e}$ and $U_{d}$ are plotted versus the coating thickness $t$ in Fig. 10. Although the stored elastic strain energy per unit of volume, $U_{e}$, decreases with increasing coating thickness, the elastic strain energy released by debonding per unit area of interface, $U_{d}$, increases with increasing coating thickness. This suggests that the extent of debonding increases with increasing coating thickness.

\section{B. Diffraction stress analysis}

By (x-ray) diffraction stress analysis, laterally and depth-averaged values for the stress are determined; see Eq. (9). In the presence of nonuniform stresses, the (x-ray) intensity weighted average stress depends on the linear absorption coefficient and the diffraction geometry [cf. Eq. (7)]. Adopting the freestanding column model

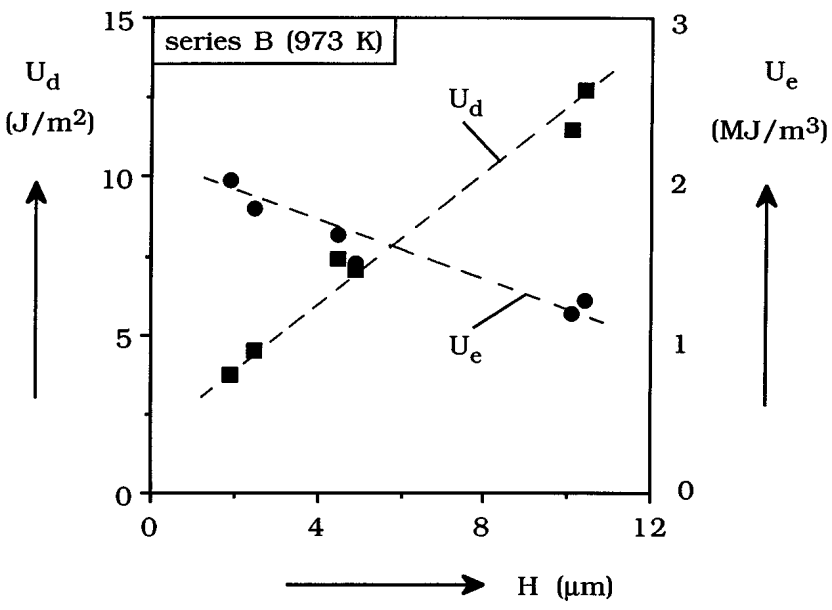

FIG. 10. The elastic strain energy $U_{e}$ stored in the CVD (at $973 \mathrm{~K}$ ) TiN coatings on Mo substrates of series B and the elastic strain energy $U_{d}$ released by debonding at the coating/substrate interface both as a function of the column height $H$ (taken as the coating thickness $t$, cf. Table I). $U_{e}$ is an average energy per unit volume coating; $U_{d}$ is an average energy per unit of area interface between coating and substrate. (Lines are drawn only to guide the eye.)

indicated above, a prediction for the experimentally determined (x-ray) diffraction-averaged stress of the TiN coatings (see Table I) is based on the following elements: (i) the dimensions (width $L$ and height $H$ ) of the columns in the TiN coatings, which are identified with the observed mesh width of the crack pattern $s$ and the coating thickness $t$ (see Table I); (ii) full elastic accommodation of the difference in thermal shrink between coating and substrate by the (cracked) TiN coating; (iii) averaging, according to Eq. (7), of the local stresses thus calculated.

The averaged stress values thus predicted can be compared with the experimental data in Fig. 11(a) for series A and Figs. 11(b) for series B. The data shown pertain to $\left\langle\sigma_{\| /}\right\rangle_{\psi_{0}}$ as derived from the $\{531\}-\mathrm{CuK} \mathrm{K}_{\beta}$ reflection of TiN.

The predicted stresses agree with the experimentally determined stresses within experimental error for all the coatings of series A [see Fig. 11(a)]. For the same coatings it was already shown in Sec. IV.E (Fig. 6) that also an excellent agreement exists between the predicted (on the same basis) and the experimentally determined $\left\langle d_{\psi}^{H K L}\right\rangle$ versus $\sin ^{2} \psi$ curves. Hence, the description of the TiN coating as an assembly of freestanding columns in conjunction with a fully elastic accommodation of the thermal misfit imposed at the coating/substrate interface is in quantitative agreement with the experimental data.

For the coatings of series B, the predicted stress values agree with the experimental data for the thin coatings [see Fig. 11(b)]. For larger thicknesses the experimentally determined stress values are smaller than those predicted. This is explained as a result of the partial debonding of these TiN coatings (see Sec. V. A). Indeed, 


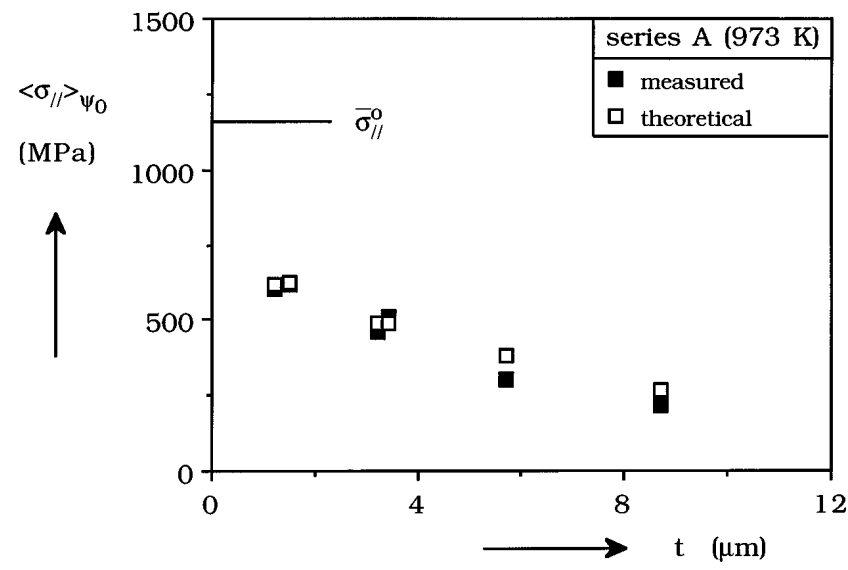

(a)

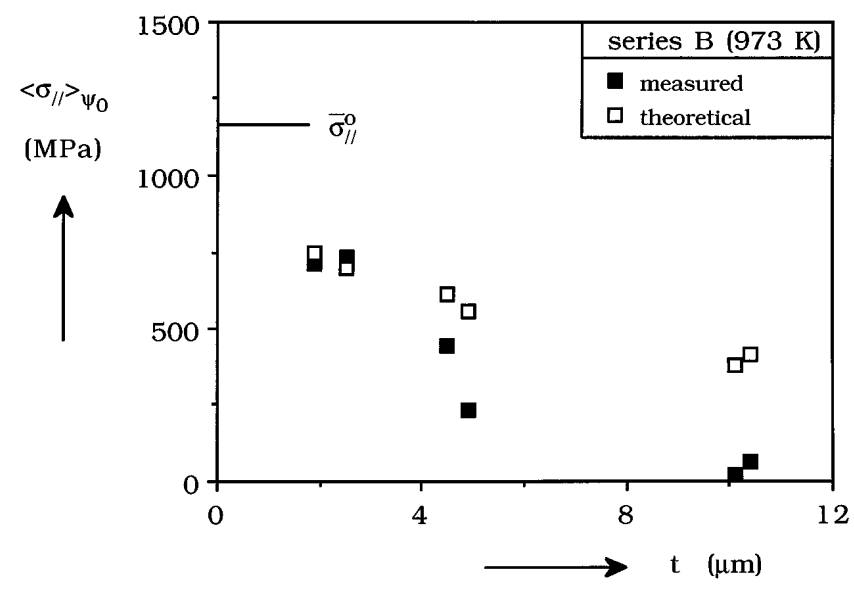

(b)

FIG. 11. Diffraction-averaged stress $\left\langle\sigma_{/ /}\right\rangle_{\psi_{0}}$ as a function of coating thickness $t$ for the CVD (at $973 \mathrm{~K}$ ) TiN coatings on Mo substrates of series A (a) and B (b); cf. Table I. The stress $\bar{\sigma}_{/ /}^{0}$ indicates the stress expected, if the thermal misfit between the coating and the substrate is taken up fully elastically by a massive and uncracked TiN coating (cf. Sec. V. A).

the adhesion of the TiN coatings of series B with the substrate is much less than for those of series A, where no delamination had occurred (cf. Sec. IV.A).

The laterally averaged stresses $\bar{\sigma}_{/ /}$[cf. Eq. (8)] are, in contrast with the diffraction-averaged stresses $\left\langle\sigma_{/ /}\right\rangle$[cf. Eq. (9)], independent of the linear absorption coefficient and the diffraction geometry. Extraction of the depth profiles of the laterally averaged stress from the diffraction data is necessary to acquire information regarding the distribution of stress over the thickness of the coating, irrespective of the measurement technique employed.

It has been demonstrated that the model adopted explains the experimental data for the diffraction-averaged stress quantitatively, if no delamination of the coating had occurred. Therefore, a prediction of the laterally averaged stress distributions for these coatings will be obtained on the same basis. In order to compare the results for the TiN coatings of different thicknesses, the laterally averaged stresses were presented as a function of the distance to the coating/substrate interface, $h$, for the TiN coatings of series A in Fig. 12. [For the TiN coatings of equal thickness (see Table I) average values were taken for the column geometry factor.]

The laterally averaged stress at the coating/substrate interface $(h=0), \bar{\sigma}_{/ /}^{0}$, is equal to the stress expected from the difference in thermal shrink between coating and substrate (cf. Sec. V.A). The stress decreases with increasing distance to the coating/substrate interface, due to relaxation by the presence of cracks. The stress gradient is relatively large near the coating/substrate interface (at the bottom of the coating) and decreases in the direction of the surface of the coating. [This illustrates that the stress gradient has a small effect on the curvature of the $\left\langle d_{\psi}^{H K L}\right\rangle$ versus $\sin ^{2} \psi$ curves. For the thin coatings the stress gradient in the near surface region is higher than for the thicker coatings, but this effect is compensated by the smaller variation of the analysis depth by tilting of the specimen as compared to the thick coatings (see also discussion with respect to Fig. 6 in Sec. IV.E).]

The experimental laterally averaged stress-depth profile can in principle be derived from a set of experimentally determined values for ( $\mathrm{x}$-ray) diffractionaveraged stresses (see discussion in Sec. II). In practice it is very difficult to obtain such a set of data. Methods based on measurements with different analysis depths, whether or not combined with stepwise removal of the surface layer, were tried but failed for the present TiN coatings; the effect of different analysis depths (by varying the Bragg angle (reflection) and wavelength)

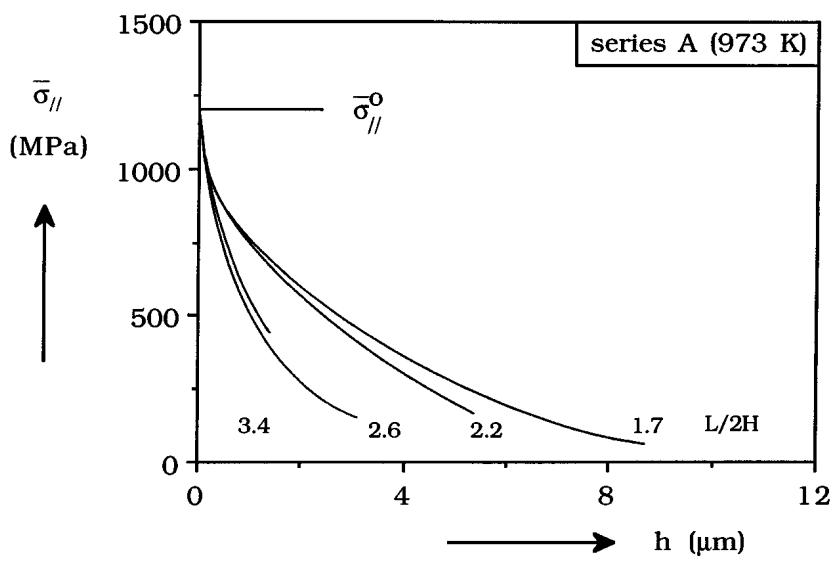

FIG. 12. Laterally averaged stress $\bar{\sigma}_{/ /}$in CVD (at $973 \mathrm{~K}$ ) TiN coatings on Mo substrates of series $\mathrm{A}$ as a function of the distance to the coating/substrate interface $h$ (cf. Fig. 8). The curves shown were obtained from the stress model for the cracked layers that provided very good agreement between the experimental and predicted diffraction-averaged stress [cf. Table I and Figs. 6 and 11(a)]. 
on the experimental data was too small for an accurate analysis of the stress distribution, and during successive removals of sublayers crumbling of the TiN coating occurred.

From the analysis in this paper, it follows that determination in a direct way by diffraction analysis of laterally averaged stress-depth profiles in surface layers is possible only if a comprehensive set of sufficiently divergent data is available, which is seldom the case in practice. However, the following indirect route has been shown to be a good alternative: (i) determine a set of diffraction-averaged stress values and (ii) develop a model for the lateral and depth variations of the stress in the surface layer that leads to quantitative agreement with the experimental results.

\section{CONCLUSIONS}

(1) The value of the stress components obtained by diffraction from surface layers exhibiting principal stress components varying on a microscopic scale can be conceived usefully as laterally averaged stresses [Eq. (9)] intensity weighted over the diffracting volume [Eqs. (6) and (10)].

(2) Direct evaluation from diffraction data of the depth profile of the laterally averaged stress in a surface layer exhibiting a nonuniform triaxial state of stress is often not practical. An advantageous approach involves development of a model for the nonuniform stresses in the surface layer which leads to a quantitative agreement between calculated and experimental values of the diffraction-averaged stresses.

(3) In TiN coatings prepared by CVD on Mo substrates large tensile stresses develop on cooling from the deposition temperature to room temperature as a consequence of the difference in thermal shrink between coating and substrate. Cracks arise because, locally at irregularities, the fracture strength is exceeded by the developing stress. The mesh width of the crack pattern depends on both the imposed stress at the layer/substrate interface and the coating thickness. The dependence is found by minimizing the sum of the stored elastic strain energy and the fracture surface energy.

(4) Values of diffraction-averaged stress were calculated by conceiving the cracked layers as assemblies of freestanding columns, with a width equal to the average crack distance and a height equal to the coating thickness, and by assuming full elastic accommodation of the thermal misfit imposed at the column/substrate interfaces. These calculated values agree well with the experimental data for a series of specimens. In those cases where the experimentally determined stress is significantly smaller than the calculated stress, partial debonding of the TiN coatings had occurred due to poor adhesion between coating and substrate. The extent of debonding increases with coating thickness.
(5) This work in addition yielded values for the following material properties of TiN: Poisson's ratio, $\nu=0.23 \pm 0.02 ;$ surface energy, $\gamma=8.2 \mathrm{~J} / \mathrm{m}^{2}$, and fracture toughness, $K_{c}=2.6 \mathrm{MPa} \cdot \mathrm{m}^{1 / 2}$.

\section{ACKNOWLEDGMENTS}

The authors are indebted to ing. W. Hendriks and Mr. G. Verspui of Philips Centre for Manufacturing Technology, Eindhoven, The Netherlands, for provision of CVD facilities and assistance with the CVD experiments. We are indebted to our former student Ir. E. G. Scheepers, who contributed to this work. Financial support of the "Foundation of Fundamental Research on Matter (FOM)" is gratefully acknowledged.

\section{REFERENCES}

1. W. D. Nix, Metall. Trans. A 20A, 2217 (1989).

2. R.W. Hoffman, Thin Solid Films 89, 155 (1982).

3. M. Murakami, T. Kuan, and I. A. Blech, in Treatise on Mater. Sci. and Technol., edited by K. N. Tu and R. Rosenberg (Academic Press, New York, 1982), Vol. 24, pp. 163, 210.

4. F. M. D'Heurle, Int. Mater. Rev. 34, 53 (1989).

5. A. G. Evans, M.D. Dory, and M. S. Hu, J. Mater. Res. 3, 1043 (1988).

6. W. G. Sloof, H. J. M. Rijpkema, R. Delhez, Th.H. de Keijser, and E. J. Mittemeijer, Surf. Eng. 3, 59 (1987).

7. W. G. Sloof, R. Delhez, Th.H. de Keijser, and E. J. Mittemeijer, J. Mater. Sci. 22, 1701 (1987).

8. W. G. Sloof, M. A. J. Somers, R. Delhez, Th.H. de Keijser, and E. J. Mittemeijer, in Residual Stresses in Science and Technology, edited by E. Macherauch and V. Hauk (Deutsche Gesellschaft für Metallkunde, Oberursel, 1987), Vol. 1, pp. 493, 500.

9. R. Delhez, Th.H. de Keijser, and E. J. Mittemeijer, Surf. Eng. 3, 331 (1987).

10. M. A. J. Somers and E. J. Mittemeijer, Metall. Trans. A 21A, 189 (1990).

11. S. Komiya, N. Umezu, and C. Hayashi, Thin Solid Films 63, 341 (1979).

12. T. A. Mäntylä, P. J. Helevirta, T. T. Lepistö, and P. T. Siitonen, Thin Solid Films 126, 275 (1985).

13. P. A. Dearnley, Surf. Eng. 1, 43 (1985).

14. E. Brozeit and H. M. Gabriel, Z. Werkstofftech. 11, 31 (1980).

15. I. Yoshizawa and K. Kamada, J. Nucl. Mater. 122\&123, 1309 (1984).

16. I. C. Noyan and J. B. Cohen, in Residual Stress-Measurement by Diffraction and Interpretation (Springer-Verlag Inc., New York, 1987), p. 121.

17. V. Hauk and E. Macherauch, in Adv. X-Ray Anal., edited by J. B. Cohen et al. (Plenum Press, New York, 1984), Vol. 27, pp. $81,99$.

18. H. Behnken and V. Hauk, Z. Metallk. 77, 620 (1986).

19. B. Eigenmann, B. Scholtes, and E. Macherauch, Mater.-wiss. u. Werkstofftech. 21, 257 (1990).

20. M. G. Moore and W.P. Evans, Trans. Soc. Automotive Eng. (SAE) 66, 340 (1958).

21. G. F. Bastin, H. J. M. Heijligers, and F. J. J. van Loo, Scanning 8, 45 (1986).

22. C. R. Hubbard, J. Appl. Crystallogr. 16, 285 (1983).

23. C. S. Barrett and T. B. Massalski, in Structure of Metals (Pergamon Press, Oxford, England, 1980), p. 204.

24. R. Kieffer and F. Beneskovsky, in Hartstoffe (Springer, Wien, Austria, 1963), p. 303. 
25. A. J. Perry, Thin Solid Films 193/194, 463 (1990).

26. X. Jiang, M. Wang, K. Schmidt, E. Dunlop, J. Haupt, and W. Gissler, J. Appl. Phys. 69, 3053 (1991).

27. D. S. Stone, K. B. Yoder, and W. D. Sproul, J. Vac. Sci Technol. A 9, 2543 (1991).

28. J. A. Sue, Surf. Coating Technol. 54/55, 154 (1992).

29. J. Birkhölzer and V. Hauk, Härterei-Tech. Mitt. 48, 25 (1993).

30. J-E. Sundgren, Thin Solid Films 128, 21 (1985).

31. A. J. Perry, Thin Solid Films 170, 63 (1989).

32. W. Kress, P. Roedhammer, H. Bilz, W.D. Teuchert, and A. N. Christensen, Phys. Rev. B 17, 111 (1978).

33. F. Bollenrath, V. Hauk, and E.H. Müller, Z. Metallk. 58, 76 (1967).

34. S. Nagakura, T. Kusunoki, F. Kakimoto, and Y. Hirosutu, J. Appl. Crystallogr. 8, 65 (1975).

35. L. Wolff, G. F. Bastin, and H. Y. M. Heijligers, Solid State Ionics 16, 105 (1977).

36. C. J. Smithells and E. A. Brandes, in Metals Reference Book, 5th ed. (Butterworths \& Co. Ltd., London, England, 1976), p. 100.

37. Y.S. Touloukian, R. K. Kirby, R.E. Taylor, and T. Y.R. Lee, in Thermophysical Properties of Matter, Thermal Expansion-Nonmetallic Solids (IFI/Plenum, New York, Washington, DC, 1977), Vol. 13, pp. 1147, 1151.

38. Y.S. Touloukian, R. K. Kirby, R.E. Taylor, and P.D. Desai, in Thermophysical Properties of Matter, Thermal Expansion-Metallic Elements and Alloys (IFI/Plenum, New York, Washington, DC, 1976), Vol. 12, pp. 208, 216.

39. L. E. Toth, in Transition Metal Carbides and Nitrides (Academic Press, New York, 1971), pp. 169, 174.

40. B. R. Lawn, in Fracture of Brittle Solids, 2nd ed. (Cambridge University Press, Cambridge, England, 1993), pp. 1, 15, and 55.

41. H. J. Frost and M. F. Ashby, in Deformation-Mechanism Maps, The Plasticity and Creep of Metals and Ceramics (Pergamon Press, Oxford, England, 1982), pp. 80, 83.

42. W. G. Sloof, Ph.D. Thesis (Delft University Press, The Netherlands, 1996).

43. B. J. Aleck, J. Appl. Mech. 16, 118 (1949).

44. I. A. Blech and A. A. Levi, J. Appl. Mech. 48, 442 (1981).

45. J. J. Blech and Y. Kantor, Computers and Structures 18, 609 (1984).

46. Z. Suo, J. Vac. Sci. Technol. A 11, 1367 (1993).

47. M. S. Hu, M. D. Thouless, and A. G. Evans, Acta Metall. 36, 1301 (1988).

48. M.D. Drory, M.D. Thouless, and A. G. Evans, Acta Metall. 36, 2019 (1988).

49. M. S. Hu and A. G. Evans, Acta Metall. 37, 917 (1989).

50. J. W. Hutchinson and Z. Suo, Adv. Appl. Mech. 29, 63 (1992).

51. M. T. Laugier, J. Mater. Sci. Lett. 2, 419 (1983).

52. A.E.H. Love, in A Treatise on the Mathematical Theory of Elasticity, 4th ed. (University Press, Cambridge, England, 1952), pp. $74,91$.

53. S.P. Timoshenko and J.N. Goodier, in Theory of Elasticity, 3rd ed. (McGraw-Hill, Tokyo, Japan, 1982), pp. 1, 14, and 246.

54. C. M. van Baal, Phys. Status Solidi A 77, 521 (1983).

55. I. C. Noyan, Metall. Trans. A 14A, 1907 (1983).

\section{APPENDIX A: MECHANICAL EQUILIBRIUM CONDITIONS FOR STRESS COMPONENTS IN SURFACE LAYERS}

Consider a body at rest subjected to external forces and a plane (surface) within the body. Applying the equations of motion (cf., e.g., Refs. 52 and 53) to the portion $P$ of the body at one side of the plane (neglecting the influence of body forces, e.g., gravitation) results in the following equilibrium conditions:

$$
\begin{array}{r}
\oiint_{P}[\sigma] \mathbf{n} d S=\underline{0}, \\
\oiint_{P}[\sigma] \mathbf{n} \times \mathbf{r} d S=\underline{0},
\end{array}
$$

where $[\sigma]$ is the local stress tensor at area $d S, \mathbf{n}$ the normal of the area $d S$, and $\mathbf{r}$ is the position vector of the center of area $d S$ with respect to the origin of the coordinate system. The integration is taken over the entire surface of the portion $P$ of the body.

These sets of equations provide mechanical equilibrium conditions for the stress (tensor) components at any plane in a body at rest. Applying them to a layer/substrate assembly (as depicted in Fig. 2), where no external forces act upon, results in the following prescriptions for the laterally averaged stress components $\bar{\sigma}_{13}, \bar{\sigma}_{23}$, and $\bar{\sigma}_{33}$ (cf. Sec. II). Equation (A2) implies that the stress tensor is symmetrical, i.e., $\bar{\sigma}_{i j}=\bar{\sigma}_{j i}$. Consider a plane in the layer parallel to the surface at depth $z$, thus with normal $\mathbf{n}=(0,0,1)$. Then [using Eq. (A1)]

$$
\begin{aligned}
& \oiint_{P} \sigma_{13} d x d y=0 \longrightarrow \bar{\sigma}_{13}=0 \\
& \oiint_{P} \sigma_{23} d x d y=0 \longrightarrow \bar{\sigma}_{23}=0 \\
& \oiint_{P} \sigma_{33} d x d y=0 \longrightarrow \bar{\sigma}_{33}=0 .
\end{aligned}
$$

These results can also be obtained from the general equations of mechanical equilibrium in a body ${ }^{54}$ and have been the subject of debate. ${ }^{55}$

The above consideration does not lead to a condition for the shear stresses $\sigma_{12}$ (or $\left.\bar{\sigma}_{12}\right)$ in the layer. The shear stress $\sigma_{12}$ is defined as the ratio of the shear strain $\epsilon_{12}$ and the shear modulus $G$ :

$$
\sigma_{12} \equiv \frac{\epsilon_{12}}{G} \text { with } \epsilon_{12} \equiv \frac{\partial u}{\partial y}+\frac{\partial v}{\partial x},
$$

where $u$ and $v$ are the local displacements in the $x$ and $y$ directions at point $(x, y)$, respectively.

In general, if the condition that for any value of $z$

$$
\bar{\sigma}_{12}=0 \leftrightarrow \oiint_{P} \sigma_{12} d x d y=0
$$

has to hold, then using Eq. (A6) it must hold at any value of $z$ that

$$
\oiint_{P} \epsilon_{12} d x d y=0 .
$$

In practice, this condition is often satisfied. An example is the case of a misfit between layer and substrate that is isotropic in the interfacial plane and (partially or completely) elastically taken up by the layer. Such a misfit $\epsilon_{m}$ can occur as a result of a difference in thermal shrink between layer and substrate according to:

$$
\boldsymbol{\epsilon}_{m}=\int_{T_{1}}^{T_{2}}\left[\alpha_{l}(T)-\alpha_{s}(T)\right] d T,
$$


where $\alpha_{l}(T)$ and $\alpha_{s}(T)$ denote the temperature dependent thermal expansion coefficients of the layer and the substrate, respectively. If the thermal expansion coefficients of both layer and substrate are isotropic or at least independent of the direction in the interfacial plane, then the misfit $\epsilon_{m}$ is a constant, say $K$. If this misfit is fully taken up by the layer at the interface with the substrate (i.e., the substrate is considered rigid), the strain in the layer at the interface with the substrate obeys:

$$
\begin{aligned}
& \epsilon_{11}=\epsilon_{22}=\epsilon_{m}=K \text { with } \\
& \epsilon_{11}=\frac{\partial u}{\partial x} \text { and } \epsilon_{22}=\frac{\partial v}{\partial y}
\end{aligned}
$$

It now follows that $u=K x$ and $v=K y$. Hence, using Eq. (A6), $\epsilon_{12}=0$ and $\sigma_{12}=0$ at any point $(x, y)$ of the interfacial plane. Consequently, no shear stresses $\sigma_{12}$ act upon the lateral planes of the layer. So, indeed for any $z$ Eq. (A7) holds: $\bar{\sigma}_{12}=0$ and thus also $\left\langle\sigma_{12}\right\rangle=0$; cf. Eq. (1) or Eq. (3).

\section{APPENDIX B: EXTRACTION OF THE DEPTH PROFILE OF THE LATERALLY AVERAGED STRESS FROM DIFFRACTION-AVERAGED STRESSES}

The depth profile of the laterally averaged stress of a surface layer can be retrieved in principle from a set of experimentally determined values of diffractionaveraged stresses (see for discussion Sec. II).

A polynomial of degree $n$ is taken for the laterally averaged stress as a function of the distance to the coating/substrate interface $h$ :

$$
\bar{\sigma}_{/ /}(h)=\sum_{k=0}^{n} a_{k} h^{k},
$$

where $h=t-z$ and $0 \leqslant h \leqslant t$ (cf. Fig. 8). The coefficient $a_{0}$ of the polynomial denotes the laterally averaged stress at the interface $(h=0), \bar{\sigma}_{/ /}^{0}$. The coefficients $a_{k}$ of the polynomial can be determined from the above-mentioned set of experimental data: $\left\langle\sigma_{\| /}\right\rangle_{p}$ [with $p=1(1) q$ where $q$ is the number of data $(q \geqslant n)]$.

The laterally averaged stress is related to the diffraction-averaged stress according to Eq. (9), which reads:

$$
\left\langle\sigma_{/ /}\right\rangle_{p}=\frac{\int_{0}^{t} \bar{\sigma}_{/ /}(z) \exp (-\mu \kappa z) d z}{\int_{0}^{t} \exp (-\mu \kappa z) d z},
$$

where $\mu$ is the linear absorption coefficient, $\kappa$ indicates the geometry factor of the diffractometer, and $z$ represents the distance to the surface. It is noted that $\left\langle\sigma_{\| /}\right\rangle_{p}$ depends on $\psi$ and $\theta$ through $\kappa$ [cf. Eqs. (4a) and (4b)].
Substitution of Eq. (B1), with $h=t-z$, yields:

$$
\left\langle\sigma_{\| /}\right\rangle_{p}=\frac{\int_{0}^{t} \sum_{k=0}^{n} a_{k}(t-z)^{k} \exp (-\mu \kappa z) d z}{\int_{0}^{t} \exp (-\mu \kappa z) d z} .
$$

With the binomial of Newton:

$$
(t-z)^{k}=\sum_{l=0}^{k}\left(\begin{array}{l}
k \\
l
\end{array}\right)(-1)^{l} t^{k-l} z^{l},
$$

Eq. (B3) becomes:

$$
\begin{aligned}
\left\langle\sigma_{\|}\right\rangle_{p}= & \sum_{k=0}^{n} a_{k} \sum_{l=0}^{k}\left(\begin{array}{l}
k \\
l
\end{array}\right) \\
& \times(-1)^{l} t^{k-l} \frac{\int_{0}^{t} z^{l} \exp (-\mu \kappa z) d z}{\int_{0}^{t} \exp (-\mu \kappa z) d z}
\end{aligned}
$$

Using

$$
\begin{aligned}
\int_{0}^{t} z^{l} \exp (-\mu \kappa z) d z= & -\left.\frac{z^{l}}{\mu \kappa} \exp (-\mu \kappa z)\right|_{0} ^{t} \\
& +\frac{l}{\mu \kappa} \int_{0}^{t} z^{l-1} \exp (-\mu \kappa z) d z
\end{aligned}
$$

Eq. (B5) transforms into

$$
\begin{array}{r}
\left\langle\sigma_{\| /}\right\rangle_{\psi_{0}, t_{p}}=a_{0}+\sum_{k=1}^{n} a_{k} \sum_{l=1}^{k}\left(\begin{array}{c}
k \\
l
\end{array}\right)(-1)^{l} t_{p}^{k-l} \\
\times\left[\frac{-\exp \left(-\mu \kappa t_{p}\right)}{1-\exp \left(-\mu \kappa t_{p}\right)} \sum_{m=0}^{l-1}\left(\frac{t_{p}^{l-m}}{(\mu \kappa)^{m}} \frac{l !}{(l-m) !}\right)\right. \\
\left.+\frac{l !}{(\mu \kappa)^{l}}\right] .
\end{array}
$$

The following recurrence relation can be derived from Eq. (B7):

$$
\begin{array}{ll}
T_{p k}=t^{k} \sum_{l=1}^{k}\left(\begin{array}{l}
k \\
l
\end{array}\right)(-1)^{l}\left[l b T_{p(l-1)}-c\right] & \text { for } k \geqslant 1 \\
T_{p k}=1 & \text { for } k=0
\end{array}
$$

$$
\text { where } b=\frac{1}{\mu \kappa t} \quad \text { and } \quad c=\frac{\exp (-\mu \kappa t)}{1-\exp (-\mu \kappa t)}
$$

(Note that for $t \rightarrow 0, T_{p k} \rightarrow 0$ for $k \geqslant 1$.) Thus Eq. (B7) can be rewritten as:

$$
\left\langle\sigma_{\|}\right\rangle_{p}=\sum_{k=0}^{n} a_{k} T_{p k}
$$


The factor $T_{p k}$ represents all the experimental parameters that can be varied to obtain different values for $\left\langle\sigma_{\|}\right\rangle_{p}$, viz. the coating thickness $t$, the attenuation coefficient $\mu$, and the diffraction geometry factor $\kappa$, which depends on the Bragg angle $\theta$ (reflection) and the tilt angle $\psi$ [cf. Eqs. (4a) and (4b)].

From a set of $q$ independent experimental data for $\left\langle\sigma_{\| /}\right\rangle_{p}, q$ polynomial coefficients $a_{k}$ can be solved in principle. However, in practice an estimate can often be made in advance for the stress at the interface $(h=$ $0), \bar{\sigma}_{/ /}^{0}$, which is equal to $a_{0}$. Then, $q+1$ polynomial coefficients $a_{k}$ can be determined. In many cases a polynomial of degree 3 will be sufficient to describe the stress-depth profile. Then, if more than three independent data for $\left\langle\sigma_{\| /}\right\rangle_{p}$ are available (i.e., $q>n$ ), the values for $a_{k}$ [with $k=0(1) n$ ] can be obtained, applying a least square fitting procedure.

\section{APPENDIX C: FRACTURE OF A BRITTLE COATING BY RESIDUAL TENSILE STRESSES}

Consider a brittle coating exhibiting a biaxial state of residual tensile stress characterized by two equal principal stresses $\left(\sigma_{11}=\sigma_{22} \equiv \sigma_{/ /}\right)$which are parallel to the surface. If the stress $\sigma_{/ /}$exceeds the fracture strength $\sigma_{f}$ of the brittle coating ("brittle" implies here that no plastic deformation takes place), the coating fractures by development of cracks extending from the surface to the interface with the substrate and the coating then is constituted of a number of "islands". Such a coating can be conceived as an arrangement of freestanding columns (cf. Sec. V.A. and Figs. 3 and 8). Then the column width $L$ and the column height $H$ correspond with the mesh width $s$ of the crack pattern and the coating thickness $t$, respectively.

The extent to which crack development takes place can be derived from the following energy balance (see Griffith energy-balance concept ${ }^{40}$ ). Crack development occurs as long as the decrease of the total elastic strain energy, $U_{e}$, is larger than the increase of the total fracture-surface energy, $U_{s}$. Hence, crack formation is governed by the energy sum $U$ according to

$$
U=U_{e}+U_{s}
$$

and crack development requires $\Delta U=\Delta U_{e}+\Delta U_{s}<$ 0 . In the following it will be assumed that the elastic energy of the substrate is negligible or does not change during fracture of the coating, then it can be ignored in the calculations [cf. Eq. (C4)]. The stored elastic strain energy per unit of volume can now be calculated using the stress distributions $\sigma_{i j}$ (with $i, j=1,2$, or 3 ) provided by the freestanding column model for a certain stage of crack formation, as described in Sec. V.A. Defining $U_{e}$ as an average energy quantity per unit volume, it follows ${ }^{53}$ :

$$
\begin{aligned}
U_{e}= & \frac{1}{2 E}\left(\frac{L}{2 H}\right)^{-2} \int_{u=0}^{L / 2 H} \int_{v=0}^{L / 2 H} \int_{w=0}^{1}\left(\sigma_{11}^{2}+\sigma_{22}^{2}+\sigma_{33}^{2}\right) \\
& -2 \nu\left(\sigma_{11} \sigma_{22}+\sigma_{22} \sigma_{33}+\sigma_{11} \sigma_{33}\right) \\
& +2(1+\nu)\left(\sigma_{12}^{2}+\sigma_{13}^{2}+\sigma_{23}^{2}\right) d u d v d w, \quad(\mathrm{C} 2)
\end{aligned}
$$

where the coordinates $u, v$, and $w$ are related to the coordinates $x, y$, and $z$ as follows: $u=x / H, v=y / H$, and $w=z / H$. Defining the fracture-surface energy $U_{s}$ also as an average energy quantity per unit of volume, it follows:

$$
U_{s}=4 m \gamma \frac{1}{L},
$$

where $\gamma$ denotes the surface energy per unit area, $L$ indicates the mesh width of the crack pattern, and $m$ is a constant depending on the shape of the cells in the crack pattern (e.g., for rectangular cells $m=1$ ).

For constant thickness $t(=H)$ of the coating, both $U_{e}$ and $U_{s}$ depend on the mesh width of the crack pattern $s(=L)$. Crack formation continues until an equilibrium (i.e., minimal) value for $U$ [cf. Eq. (C1)] occurs, which corresponds with an equilibrium value for $L$. Hence, this equilibrium value for $L$ can be obtained from:

$$
\frac{\partial U}{\partial L}=0 \longrightarrow \frac{\partial U_{e}}{\partial L}+\frac{\partial U_{s}}{\partial L}=0 .
$$

In the absence of cracks, the stress is constant throughout the coating (cf. Sec. V.A.). In the presence of cracks, this level of stress corresponds only with the stress at the coating/substrate interface; it will be denoted as $\bar{\sigma}_{/ /}^{0}$ (cf. Sec. V.B). Applying the column model and using Eq. (C2), the elastic strain energy in the cracked coating can be calculated and expressed in terms of $\bar{\sigma}_{/ /}^{0}$. Using Poisson's ratio of TiN ( $\nu=0.2$, cf. Sec. IV.C), the results of such calculations for values of the column geometry factor $(L / 2 H)$ between 1 and 6 are shown in Fig. 13. The curve of $U_{e}$ versus $L / 2 H$ can be well approximated by:

$$
\begin{aligned}
U_{e}= & (1-\nu)\left(\frac{\left(\bar{\sigma}_{/ I}^{0}\right)^{2}}{E}\right) \\
& \times\left[1-\exp \left(-0.27 \frac{L}{2 H}\right)\right] .
\end{aligned}
$$

Then, the equilibrium mesh width $L_{\mathrm{eq}}$ follows by substitution of Eqs. (C3) and (C5) into Eq. (C4):

$$
\begin{aligned}
0.27(1-\nu) \frac{\left(\bar{\sigma}_{/ /}^{0}\right)^{2}}{E} \frac{L_{\mathrm{eq}}}{2 H} \exp ( & \left.-0.27 \frac{L_{\mathrm{eq}}}{2 H}\right) \\
& -4 m \gamma \frac{1}{L_{\mathrm{eq}}}=0 .
\end{aligned}
$$




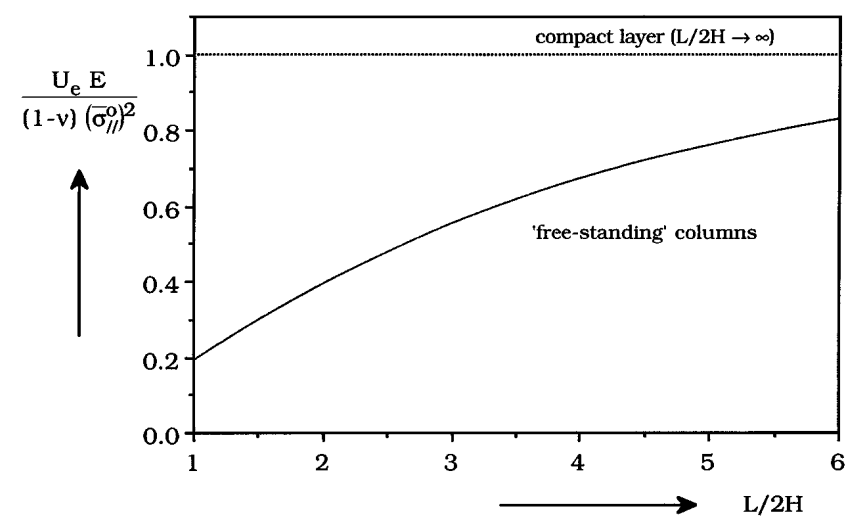

FIG. 13. Average elastic strain energy per unit volume coating, $U_{e}$ [cf. Eq. (C1)], relative to the elastic strain energy of a massive and uncracked coating, $(1-v)\left(\bar{\sigma}_{/ /}^{0}\right)^{2} / E$, as a function of the column geometry factor $L / 2 H$; calculated for TiN $[\nu=0.19(8)]$. The stress $\bar{\sigma}_{/ /}^{0}$ indicates the stress expected if the thermal misfit between the coating and the substrate is taken up fully elastically by a massive and uncracked coating (cf. Sec. V.A).

The relation between $L_{\mathrm{eq}}$ and $H$ is provided implicitly but unambiguously by Eq. (C6a); it can be calculated numerically. For practical purposes the relation between $L_{\text {eq }}$ and $H$ can be given in an explicit but approximate way, as follows. Because the curvature of the curve of
$U_{e}$ versus $L / 2 H$ in the range of $L / 2 H$ values considered here is rather small (see Fig. 13), a fair approximation for Eq. (C5) is obtained by linearizing $U_{e}$, in our case at $L / 2 H=3.5$ (which is in the middle of the considered range of $L / 2 H$ values). Thus $U_{e} \approx[0.244$ $+0.105(L / 2 H)](1-v)\left(\bar{\sigma}_{/ /}^{0}\right)^{2} / E$. Substitution of this expression for $U_{e}$ and Eq. (C3) into Eq. (C4) leads to:

$$
\left(\bar{\sigma}_{/ /}^{0}\right)^{2} L_{\mathrm{eq}}^{2}=\frac{76}{1-v} m \gamma E H
$$

or

$$
\bar{\sigma}_{/ /}^{0} L_{\mathrm{eq}}=K_{c} \sqrt{\frac{38 m H}{1-v}},
$$

where $K_{c}$ is the so-called fracture toughness of the coating material:

$$
K_{c}=\sqrt{2 \gamma E} .
$$

If for a series of cracked coatings with varying coating thickness the stress $\bar{\sigma}_{/ /}^{0}$ and the shape factor $m$ of the crack pattern are constant, then a plot of $L_{\text {eq }}^{2}$ versus $H$ should provide a straight line. From its slope a value for the surface energy can be derived, provided that Young's modulus $E$ and Poisson's ratio $\nu$ are known. 